\title{
A CONSTRUCTION OF REFLECTING BARRIER BROWNIAN MOTIONS FOR BOUNDED DOMAINS
}

\author{
Masatoshi FUKUSHIMA
}

(Received February 20, 1967)

\section{Introduction}

Let $D$ be an arbitrary bounded domain of the $N$-dimensional Euclidean space $R^{N}$.

We will call a function $G_{a}(x, y)(\alpha>0, x, y \in D, x \neq y)$ a (continuous) resolvent density on $D$ if the following conditions are satisfied:

(G. 1) $\quad G_{a}(x, y) \geqq 0, \quad \alpha>0, \quad x, y \in D, \quad x \neq y$.

(G. 2) $\quad \alpha \int_{D} G_{w}(x, y) d y \leqq 1, \quad \alpha>0, \quad x \in D .{ }^{1)}$

(G. 3) $G_{a}(x, y)-G_{\beta}(x, y)+(\alpha-\beta) \int_{D} G_{a}(x, z) G_{\beta}(z, y) d z=0$,

$\alpha, \quad \beta>0, \quad x, \quad y \in D, \quad x \neq y$.

(G. 4) For fixed $\alpha>0, G_{s}(x, y)$ is continuous in $(x, y)$ on $D \times D$ off the diagonal.

A resolvent density on $D$ is called conservative if the equality holds in (G.2) for all $\alpha>0$ and all $x \in D$.

In this paper, we will construct a conservative resolvent density on $D$ and show that it determines a diffusion process (that is, a strong Markov process having continuous trajectories) which takes values in a natural enlarged state space $D^{*}$. When the relative boundary $\partial D$ of $D$ is sufficiently smooth, our diffusion process is shown (Theorem 6) to be the well known reflecting barrier Brownian motion on $D \cup \partial D$. For this reason, our process for an arbitrary $D$ may be considered the reflecting barrier Brownian motion in an extended sense.

A function $p(t, x, y), t>0, x, y \in D$, will be called a (continuous) transition density on $D$, if it satisfies the following conditions:

(T. 1) $\quad p(t, x, y) \geqq 0, t>0, x, y \in D$.

1) $d y$ denotes the Lebesgue measure on $D$. 


$$
\int_{D} p(t, x, y) d y \leqq 1, t>0, x \in D \text {. }
$$

(T. 3) $\quad p(t+s, x, y)=\int_{D} p(t, x, z) p(s, z, y) d z, t, s>0, x, y \in D$.

(T. 4) $\quad p(t, x, y)$ is continuous in $(t, x, y) \in(0,+\infty) \times D \times D$.

A transition density for which the equality holds in (T. 2) for all $t>0$ and all $x \in D$ will be called conservative.

Let $p^{0}(t, x, y)$ be the transition density corresponding to the absorbing barrier Brownian motion on $D^{2}$. Set

$$
G_{a}^{0}(x, y)=\int_{0}^{+\infty} e^{-\infty t} p^{0}(t, x, y) d t, \quad \alpha>0, \quad x, y \in D,
$$

then $G_{\alpha}^{0}(x, y)$ is a resolvent density on $D$ and can be expressed in the form,

$$
G_{a}^{0}(x, y)=\Pi_{a s}(x, y)-\widetilde{E}_{x}\left(e^{-a \tau} \Pi_{a x}\left(X_{\tau}, y\right)\right) \quad \alpha>0, x, y \in D,
$$

where,

$$
\Pi_{\infty}(x, y)=\int_{0}^{+\infty} e^{-\infty t} \frac{1}{(2 \pi t)^{N / 2}} e^{-(|x-y| 2 / 2 t)} d t, x, y \in R^{N 3)},
$$

$\widetilde{E}_{x}$ is the expectation with respect to the standard Brownian measure $\widetilde{P}_{x}, x \in D$, and $\tau$ is the first exist time from $D$ of the Brownian path $X_{t}$.

A function $u$ defined on an open set $U$ of $R^{N}$ will be called $\alpha$-harmonic on $U$ if

$\left(\alpha-\frac{1}{2} \Delta\right) u(x)=0, x \in U$, where $\Delta$ is the Laplacian; $\Delta=\sum_{i=1}^{N} \frac{\partial^{2}}{\partial x_{i}^{2}}$. For functions $u$, $v$ on $D$, we set

$$
\begin{aligned}
& (u, v)=\int_{D} u(x) v(x) d x, \\
& \boldsymbol{D}(u, v)=\int_{D}(\operatorname{grad} u, \operatorname{grad} v)(x) d x .
\end{aligned}
$$

For each $\alpha>0$, let $\boldsymbol{H}_{a}$ be the Hilbert space formed by all $\alpha$-harmonic functions on $D$ with the following norm:

$$
\boldsymbol{D}_{a}(u, u)=\boldsymbol{D}(u, u)+2 \alpha(u, u)<+\infty .
$$

In section 2 , we shall prove the following.

\section{Theorem 1.}

(i) For each $\alpha>0$ and each $x \in D$, there exists a unique $y$-function $R_{\alpha}^{x}(y)$ $=R_{o s}(x, y)$ in $\boldsymbol{H}_{a}$ such that the equation

2) cf. [8].

3) $|x-y|$ denotes the distance between $x$ and $y$. 


$$
\boldsymbol{D}\left(R_{\alpha}^{x}, v\right)+2 \alpha\left(R_{\alpha}^{x}, v\right)=2 v(x)
$$

holds for all $v \in \boldsymbol{H}_{\boldsymbol{\alpha}}$.

(ii) Set

$$
G_{\omega}(x, y)=G_{\alpha}^{0}(x, y)+R_{\alpha}(x, y), \quad \alpha>0, \quad x, y \in D .
$$

Then $G_{a}(x, y)$ is a conservative resolvent density on $D$, symmetric in $x, y \in D$.

(iii) Denote by $\boldsymbol{B}(D)($ resp. $\boldsymbol{C}(D))$ the collection of all bounded measurable (resp. bounded continuous) functions on $D$. The operator $G_{a}$ defined by

$$
G_{o} f(\cdot)=\int_{D} G_{o}(\cdot, y) f(y) d y, \quad f \in \boldsymbol{B}(D),
$$

maps $\boldsymbol{B}(D)$ into $\boldsymbol{C}(D)$. Moreover, if $f \in \boldsymbol{C}(D)$, then $\lim _{\alpha \rightarrow+\infty} \alpha G_{a} f(x)=f(x), x \in D$.

(iv) Suppose that $K_{1}$ and $K_{2}$ are compact, $D_{1}$ is open and $K_{1} \subset D_{1} \subset K_{2} \subset D$. Then, $\sup _{x \in K_{1}, y \in D-K_{2}} G_{w}(x, y)$ is finite.

(v) There is a unique transition density $p(t, x, y)$ on $D$ satisfying

$$
G_{\alpha}(x, y)=\int_{0}^{+\infty} e^{-\alpha t} p(t, x, y) d t, \quad \alpha>0, \quad x, y \in D .
$$

$p(t, x, y)$ is conservative and $\int_{D} p(t, x, y) f(y) d y$ is continuous in $(t, x) \in(0,+\infty)$ $\times D$ for any $f \in \boldsymbol{B}(D)$.

When $\partial D$ is suffciently smooth, the transition density in Theorem 1 turns out to be the fundamental solution of the heat equation $\left(\frac{\partial}{\partial t}-\frac{1}{2} \Delta_{x}\right) u(t, x)$ $=0, t>0, x \in D$, with the boundary condition $\frac{\partial}{\partial n_{x}} u(t, x)=0, t>0, x \in \partial D$, where $n_{x}$ is the inner normal at the point $x \in \partial D$. Indeed, assuming that $\partial D$ is in class $C^{3}$, let us denote the latter by $\dot{p}(t, x, y), t>0, x, y \in D$. Then, it is a transition density and

$\dot{R}_{\alpha}(x, y)=\int_{0}^{+\infty} e^{-\alpha t} \dot{p}(t, x, y) d t-G_{\alpha}^{0}(x, y)$ is an $\alpha$-harmonic function in the class $\boldsymbol{C}^{1}(D \cup \partial D)$ as a function of $y^{4}$. Hence, we have only to show that $\dot{R}_{\alpha}^{x}=\dot{R}_{\alpha}(x, \cdot)$ satisfies equation (1.5). Applying the Green formula to the identity $-\frac{\partial}{\partial n_{y}} \dot{R}_{\alpha}^{x}(y)=\frac{\partial}{\partial n_{y}} G_{\alpha}^{0}(x, y), y \in \partial D$, we see that

$$
\frac{1}{2} \boldsymbol{D}\left(\dot{R_{\alpha}^{x}}, v\right)+\alpha\left(\dot{R_{\alpha}^{x}}, v\right)=\frac{1}{2} \int_{\partial D} \frac{\partial}{\partial n_{y}} G_{\alpha}^{0}(x, y) v(y) \sigma(d y)
$$
$D \cup \partial D$.

4) cf. [7]. $\boldsymbol{C}^{1}(D \cup \partial D)$ denotes the totality of continuously differentiable functions on 
holds for every $v \in \boldsymbol{C}^{1}(D \cup \partial D), \sigma(d y)$ standing for the surface Lebesgue measure of $\partial D$. The right hand side of (1.8) is the $\alpha$-harmonic function with the boundary value $v$. A usual limiting procedure leads us to the validity of (1.5) for $\dot{R}_{a}^{x}$ and for every $v \in \boldsymbol{H}_{a}{ }^{5}$.

We call a compact set $D^{*}$ a compactification of $D$ if $D^{*}$ contains $D$ as an open dense subset and the relative topology of $D$ in $D^{*}$ is equivalent to the original Euclidean topology there. In Sections 3 and 4, the following theorem will be proved.

\section{Theorem 2.}

(i) There is a compactification $D^{*}$ of $D$ such that $p(t, x, y), t>0$, of Theorem 1 is extended to $(x, y) \in D^{*} \times D$ uniquely in a certain way and the extended function (denoted again by $p(t, x, y)$ ) satisfies conditions (T. 1), (T. 2) and (T. 3) for $x \in D^{*}$ and $y \in D$.

(ii) There exists a Markov process $\boldsymbol{X}=\left\{X_{t}, P_{x}, x \in D^{*}\right\}$ possessing the following properties.

(a) For each Borel set $A$ of $D^{*}$,

$$
P_{x}\left(X_{t} \in A\right)=\int_{D \cap A} p(t, x, y) d y, t>0, \quad x \in D^{*} .
$$

(b) $\boldsymbol{X}$ is continuous;

$P_{x}\left(X_{t}\right.$ is continuous in $t$ for every $\left.t \geqq 0\right)=1, \quad x \in D^{*}$.

(c) X has the strong Markov property.

(d) The part of $\boldsymbol{X}$ on the set $D$ is the absorbing barrier Brownian motion there; for every $x \in D$ and Borel set $A$ of $D$,

$$
P_{x}\left(X_{t} \in A ; t<\tau\right)=\int_{A} p^{0}(t, x, y) d y, \quad t>0,
$$

$\tau$ being the first exit time from $D$.

(e) There exists a Borel subset $D_{1}^{*}$ of $D^{*}$ containing $D$ such that

$$
\begin{array}{ll}
P_{x}\left(X_{0}=x\right)=1, & x \in D_{1}^{*}, \\
P_{x}\left(X_{0}=x\right)=0, & x \in D^{*}-D_{1}^{*} .
\end{array}
$$

Moreover $\boldsymbol{X}$ is conservative on $D_{1}^{*} ; P_{x}\left(X_{t} \in D_{1}^{*}\right.$ for every $\left.t \geqq 0\right)$ $=1, \quad x \in D_{1}^{*}$.

5) For $v \in \boldsymbol{H}_{\alpha}$, we can find a sequence of functions $v_{n} \in \boldsymbol{C}^{1}(D \cup \partial D)$ which converges to $v$ with respect to the norm $\sqrt{ } \overline{\boldsymbol{D}(v, v)+2 \alpha(v, v)}$. The boundary function of $v_{n}$, then, converges to that of $v$ (which is determined by $v, \sigma$-almost everywhere on $\partial D$ ) in $\mathbf{L}^{2}(\sigma)$ sense. 
Let $D^{*}$ be the completion of $D$ of the Martin-Kuramochi type with respect to the resolvent density $G_{1}(x, y)$ of Theorem $1^{6}$. In Section 3 , we will show that this $D^{*}$ satisfies condition (i) of Theorem 2 and we will derive a right continuous strong Markov process $\boldsymbol{X}$ on $D^{*}$ satisfying the condition (ii, a). Moreover, the property (ii, d) will be verified.

We now give some conments on the completion in Theorem 2 . The first remark is that the validity of Theorem 2 (i) for our $D^{*}$ owes essentially to the conservativity of the resolvent density of Theorem 1 . The second remark is concerned with the strong Markov property of $\boldsymbol{X}$ in the theorem. D. Ray [20] proved that, under certain hypotheses, to a resolvent on a compact space corresponds a strong Markov process. One of Ray's hypotheses is that the given resolvent makes invariant the space of all continuous functions. This condition, however, is not necessarily satisfied by the resolvent (operator) induced by the density function $G_{o}(x, y)$ on the extended space $D^{*}$. Therefore, Ray's original theorem is not enough to verify the strong Markov property of our $\boldsymbol{X}$. We will reproduce the proof of $\mathrm{H}$. Kunita and $\mathrm{H}$. Nomoto [9]; they treat a wide class of Markov processes including ours. ( $T$. Watanabe pointed out that there is another nice completion for which Ray's original results can be applied in themselves. Under this completion, Theorem 2 is still valid and the conservativity of the resolvent density is irrelevant. See [11].) Third, we note that $D^{*}-D_{1}^{*}$ is the set of all branching points in Ray's sense [20] ${ }^{7}$. Finally, statements (b) and (e) imply that almost all trajectories starting from a non-branching point never contact with branching points.

In order to complete the proof of Theorem 2, we must show the continuity of trajectories of $\boldsymbol{X}$. Section 4 will be devoted to the proof of the above feature of $\boldsymbol{X}$ by a potential-theoretic method. First, $G_{1}(x, y)$ of Theorem 1 will be extended to $(x, y) \in D^{*} \times D^{*}$ and every summable 1-excessive function will be expressed as the integral of the kernel $G_{1}(x, y)$ with a unique measure on $D_{1}{ }^{*}$ (Theorem 3). Second, we will introduce the notion of the Dirichlet norm \|\|$u \|_{\boldsymbol{X}}$ of the function $u(x)=\int_{D} G_{1}(x, y) f(y) d y, x \in D^{*}, f \in \boldsymbol{B}(D)$, with respect to our process $\boldsymbol{X}$ and we will then show (Theorem 4) that the equality $\|\mid u\| \|_{\boldsymbol{X}}^{2}$ $=\int_{D}(\operatorname{grad} u, \operatorname{grad} u)(x) d x$ holds for each function of above type. This is a characteristic feature of reflecting barrier Brownian motions. Owing to the result of M. Motoo and S. Watanabe [18], this characteristic property of $\boldsymbol{X}$ permits us to conclude that, for any additive functional $A_{t}$ of $\boldsymbol{X}$ such as $E_{x}\left(A_{t}\right)=0$ and $E_{x}\left(A_{t}^{2}\right)<+\infty, x \in D^{*}, t>0$, the stochastic integral $\int \chi_{D_{1}^{*}-D} d A_{s}$ vanishes

6) cf. [12] and [13].

7) For $x \in D^{*}-D_{1}^{*}$, the life time of our path $X_{t}$ is either infinity or zero $P_{x}$-almost every-where (see Lemma 3.4 and 3.5). 
identically (Theorem 5). Here, $\chi_{D_{1}{ }^{*}-D}$ is the indicator function of $D_{1}^{*}-D$. This property of $\boldsymbol{X}$ will exclude the possibility that the trajectories of $\boldsymbol{X}$ have jumps on $D_{1}^{*}-D$ with positive probability.

Acknowledgement. K. Ito and N. Ikeda suggested me the problem treated here and encouraged me throughout the research. The analysis of the continuity of trajectories performed in $\S 3$ and $\S 4$ is in debt to valuable advices by H. Kunita and S. Watanabe. I wish to thank them all for their kindness. Thanks are due to K. Sato and T. Watanabe for their kind and useful opinion on the manuscript.

\section{Construction of resolvent density (proof of Theorem 1)}

From now on, we fix an arbitrary bounded domain $D$ of $R^{N}$. The following criterion for a function on $D$ to be $\alpha$-harmonic is easily verified and it will be frequently used in this paper.

Lemma 2.1. Let $\alpha$ be positive number. A function $u$ on $D$ is $\alpha$-harmonic, if and only if, for each ball $B$ with closure contained in $D$, it holds that

$$
u(x)=\int_{\partial B} h_{\alpha}^{B}(x, y) u(y) \sigma(d y), \quad x \in B,
$$

where $\sigma(d y)$ is the surface Lebesgue measure of $\partial B$ and $h_{\alpha}^{B}(x, y)=\frac{1}{2} \frac{\partial}{\partial n_{y}}{ }^{B} G_{\alpha}^{0}(x, y)$, $x \in B, y \in \partial B,{ }^{B} G_{a}^{0}(x, y)$ being the resolvent density defined by (1.1) for the ball $B$.

For functions $u$ and $v$ on $D$, define $\boldsymbol{D}(u, v)$ and $(u, v)$ by (1.3). Put

$$
\boldsymbol{D}_{\omega}(u, v)=\boldsymbol{D}(u, v)+2 \alpha(u, v), \quad \alpha>0 .
$$

Denote by $\boldsymbol{H}_{a}$ the space of all $\alpha$-harmonic functions $u$ satisfying $\boldsymbol{D}_{a}(u, u)<+\infty$.

Lemma 2.2. For each $\alpha>0, \boldsymbol{H}_{a}$ forms a real Hilbert space with the inner product $\boldsymbol{D}_{a}(u, v)$. Moreover, any Cauchy sequence of functions in $\boldsymbol{H}_{a}$ with respect to the norm $\sqrt{D_{a}(u, u)}$ converges on $D$ uniformly on any compact subset of $D$.

Proof. Suppose that $u_{n} \in \boldsymbol{H}_{a}, n=1,2, \cdots$, and $\boldsymbol{D}_{a}\left(u_{n}-u_{m}, u_{n}-u_{m}\right) \underset{n, m \rightarrow+\infty}{\longrightarrow} 0$. Let $K$ be any compact subset of $D$. Choose $\varepsilon>0$ smaller than the distance of $K$ with $\partial D$. Let $B_{\varepsilon}(x)$ be the ball with radius $\varepsilon$ centered at $x$ in $K$. Applying Lemma 2.1 to the $\alpha$-harmonic function $u_{n}-u_{m}$, we have

$$
\begin{aligned}
& u_{n}(x)-u_{m}(x) \\
& =\frac{1}{V_{\varepsilon}} \int_{B_{g}(x)} \eta_{a r}(|y-x|)\left(u_{n}(y)-u_{m}(y)\right) d y, \quad x \in K,
\end{aligned}
$$

where $V_{\varepsilon}$ is the volume of $B_{\varepsilon}(x),|y-x|$ is the distance between $x$ and $y$, and $\eta_{\alpha}(r)$ is a function of real $r>0$ which depends only on $\alpha>0$ and satisfies 
$0<\eta_{\infty}(r)<1$. The Schwarz inequality applied to (2.2) leads to

$$
\begin{aligned}
& \left(u_{n}(x)-u_{m}(x)\right)^{2} \leqq \frac{1}{V_{\mathrm{\varepsilon}}}\left(u_{n}-u_{m}, u_{n}-u_{m}\right) \\
& \leqq \frac{1}{2 \alpha V_{\mathrm{\varepsilon}}} \boldsymbol{D}_{a}\left(u_{n}-u_{m}, u_{n}-u_{m}\right), \quad x \in K .
\end{aligned}
$$

Thus, $u_{n}$ converges to a function $u$ on $D$ uniformly on any compact subset of $D$. By virtue of Lemma 2.1, $u$ is also $\alpha$-harmonic on $D$ and the first derivatives of $u_{n}$ converge to those of $u$ uniformly on any compact subset of $D$. On the other hand, since $u_{n}, n=1,2, \cdots$, form a Cauchy sequence with respect to the norm $\boldsymbol{D}_{a}$, one can find, for any $\varepsilon>0$, a compact subset $K \subset D$ such that

$$
\int_{D-K}\left|\operatorname{grad} u_{n}\right|^{2}(x) d x+2 \int_{D-K} u_{n}(x)^{2} d x<\varepsilon
$$

uniformly in $n$. Hence, $u \in \boldsymbol{H}_{a}$ and $\boldsymbol{D}_{s}\left(u_{n}-u, u_{n}-u\right) \underset{n \rightarrow+\infty}{\longrightarrow} 0$.

Lemma 2.3. Let $\alpha>0$ be fixed.

(i) For each $x \in D$, there exists a function $u^{(\boldsymbol{x})} \in \boldsymbol{H}_{\infty}$ uniquely such that

$$
\boldsymbol{D}_{a s}\left(u^{(x)}, v\right)=2 v(x), \text { for any } v \in \boldsymbol{H}_{a} .
$$

(ii) The function $u^{(x)}$ in (i) is a unique element of $\boldsymbol{H}_{a}$ minimizing the value of the functional $\Psi(u)=\boldsymbol{D}_{\infty}(u, u)-4 u(x)$ on $\boldsymbol{H}_{a b}$.

Proof. (i). For a fixed $x \in D$, define the linear mapping $\Phi$ from $\boldsymbol{H}_{a}$ to $R^{1}$ by $\Phi(v)=2 v(x), v \in \boldsymbol{H}_{a} . \quad \Phi$ is continuous by the latter half of Lemma 2.2. The Riesz theorem implies (i).

(ii). We have only to notice the equality $\Psi(u)=\Psi\left(u^{(x)}\right)+D_{a}\left(u-u^{(x)}\right.$, $\left.u-u^{(x)}\right), u \in \boldsymbol{H}_{a}$.

Definition 1. For $\alpha>0$ and $x, y \in D$, denote by $R_{a}^{x}(y)=R_{a}(x, y), y \in D$, the function $u^{(x)}(y)$ of Lemma 2.3.

Definition 2. Let $G_{a}^{0}(x, y)$ by the resolvent density defined by (1.1). Define the function $G_{w}(x, y), \alpha>0, x, y \in D$, by

$$
G_{a}(x, y)=G_{a}^{0}(x, y)+R_{a}(x, y) .
$$

Before examining those properties of $G_{a}(x, y)$ stated in Theorem 1, we prepare three lemmas.

An exhaustion of $D$ is a sequence of domains $D_{n}, n=1,2, \cdots$, such that the closure of $D_{n}$ is contained in $D_{n+1}$ and $D_{n}$ converges monotonically to $D$. An exhaustion $\left\{D_{n}\right\}$ of $D$ is called regular if $\partial D_{n}$ are of class $C^{3}$. 
Lemma 2.4. Let $\alpha>0$ be fixed.

(i) Any non-negative $\alpha$-harmonic function on $D$ is either identically zero on $D$ or strictly positive on $D$.

(ii) The function $w=1-\alpha G_{\alpha}^{0} 1$ is strictly positive on $D$. Moreover $w$ is the unique element in $\boldsymbol{H}_{a}$ satisfying

$$
\boldsymbol{D}_{a}(w, v)=2 \alpha(1, v) \text { for all } v \in \boldsymbol{H}_{a s} .
$$

Proof. (i). Since Lemma 2.1 implies that the value of an $\alpha$-harmonic function at any point of $D$ is a weighted volume mean on the ball centered at the point, property (i) is verified in the same manner as in the case of harmonic functions.

(ii). It is evident, by expression (1.2) of $G_{\alpha}^{0}$, that $w$ is $\alpha$-harmonic and strictly positive on $D$. In order to show identity (2.4), consider a regular exhaustion $\left\{D_{n}\right\}$ of $D$.

Put $w_{n}=\chi_{D_{n}}-\alpha^{n} G_{\alpha}^{\Omega} \chi_{D_{n}}$, where $\chi_{D_{n}}$ is the indicator function of $D_{n},{ }^{n} G_{\alpha}^{\ulcorner} \chi_{D_{n}}(x)=\int_{D_{n}}{ }^{n} G_{\alpha}^{\mathrm{n}}(x, y) d y$ and ${ }^{n} G_{\alpha}^{\mathrm{r}}(x, y)$ is the resolvent density (1. 1) for $D_{n}$. The function $w_{n}$ is $\alpha$-harmonic in $D_{n}$, converges to $w$ monotonically and (consequently) uniformly on any compact subset of $D$. On account of Lemma 2. 1, the derivatives of $w_{n}$ converge to those of $w$ on $D$. Denote by $\boldsymbol{D}_{a}^{n}(\quad, \quad)$ the integral (2.1) on $D_{n}$. Since $w_{n}$ belongs to $\boldsymbol{C}^{1}\left(D_{n} \cup \partial D_{n}\right)$, we can apply Green's formula to $w_{n}$ and $v \in \boldsymbol{H}_{a}$, obtaining $\boldsymbol{D}_{a}^{n}\left(w_{n}, v\right)=2 \alpha\left(\chi_{D_{n}}, v\right)$. This equality implies the inequality $\boldsymbol{D}_{a}^{n}\left(w_{n}, w_{n}\right)-4 \alpha\left(\chi_{D_{n}}, w_{n}\right) \leqq \boldsymbol{D}_{\alpha}^{n}(v, v)$ $-4 \alpha\left(\chi_{D_{n}}, v\right)$ for all $v \in \boldsymbol{H}_{a}$. Letting $n$ tend to infinity and using Fatou's lemma, we obtain

$$
\boldsymbol{D}_{w}(w, w)-4 \alpha(1, w) \leqq \boldsymbol{D}_{a}(v, v)-4 \alpha(1, v) .
$$

Thus, $w \in \boldsymbol{H}_{\alpha}$, and if we put, instead of $v, w+\varepsilon v$ in the inequality above, we arrive at (2.4). The proof of the uniqueness is straightforward.

Lemma 2.5. Take an exhaustion $\left\{D_{n}\right\}$ of $D$ arbitrarily. Let ${ }^{n} R_{\alpha}^{x}(y)$ and ${ }^{n} G_{o}(x, y), \alpha>0, x, y \in D_{n}$ be the functions defined by Definition 1 and Definition 2 for the domain $D_{n}$. Then, $\lim _{n \rightarrow+\infty}{ }^{n} G_{\infty}(x, y)=G_{\infty}(x, y), \alpha>0, x, y \in D, x \neq y$. Moreover, for each $x \in D$, the equality

$$
\lim _{n \rightarrow+\infty}{ }^{n} R_{\alpha}^{x}(y)=R_{\alpha}^{x}(y), \quad y \in D,
$$

holds and the convergence is uniform in $y$ on any compact subset of $D$.

Proof. Let ${ }^{n} G_{\alpha}^{0}(x, y)$ be the resolvent density defined by (1. 1) for the domain $D_{n}$. Since ${ }^{n} G_{\alpha}^{0}(x, y)$ increases to $G_{a}^{0}(x, y)$ we have only to discuss the convergence of ${ }^{n} R_{\alpha}^{x}$ to $R_{\alpha}^{x}$. 
Let us fix $x \in D$. We can assume that $x$ is in $D_{1}$. For each $D_{n}$, denote its associated $\alpha$-Dirichlet norm by $\boldsymbol{D}_{\alpha}^{n}$ and its associated Hilbert space by $\boldsymbol{H}_{\alpha}^{n}$. It is clear that, if $m<n$, the restriction of the function of $\boldsymbol{H}_{a}^{n}$ to $D_{m}$ is an element of $\boldsymbol{H}_{a}^{m}$.

If $m<n$, we have

$$
\begin{aligned}
& D_{a}^{m}\left({ }^{n} R_{\alpha}^{x}-{ }^{m} R_{\alpha}^{x},{ }^{n} R_{\alpha}-{ }^{m} R_{a}^{x}\right) \\
& =\boldsymbol{D}_{\alpha}^{m}\left({ }^{n} R_{\alpha}^{x},{ }^{n} R_{\alpha}^{x}\right)-2 \boldsymbol{D}_{\alpha}^{m}\left({ }^{m} R_{\alpha}^{x},{ }^{n} R_{\alpha}^{x}\right)+\boldsymbol{D}_{\alpha}^{m}\left({ }^{m} R_{\alpha}^{x},{ }^{m} R_{\alpha}^{x}\right) .
\end{aligned}
$$

We will apply Lemma 2.3 to each term of the last expression. The first term is not greater than $\boldsymbol{D}_{\alpha}^{n}\left({ }^{n} R_{\alpha}^{x},{ }^{n} R_{\alpha}^{x}\right)=2^{n} R_{\alpha}^{x}(x)$. The second and third terms are equal to $-4^{n} R_{\alpha}^{x}(x)$ and $2^{m} R_{\alpha}^{x}(x)$, respectively. Therefore, for each $N$, it holds that

$$
0 \leqq D_{\alpha}^{N}\left({ }^{n} R_{\alpha}^{x}-{ }^{m} R_{\alpha}^{x},{ }^{n} R_{\alpha}^{x}-{ }^{m} R_{\alpha}^{x}\right) \leqq 2\left({ }^{m} R_{\alpha}^{x}(x)-{ }^{n} R_{\alpha}^{x}(x)\right),
$$

for any $m$ and $n$ such that $N \leqq m<n$. Inequality (2.6) implies that ${ }^{n} R_{\alpha}^{x}(x)$ is non-increasing in $n$ and since ${ }^{n} R_{\alpha}^{x}(x)=\frac{1}{2} D_{\alpha}^{n}\left({ }^{n} R_{\alpha}^{x},{ }^{n} R_{\alpha}^{x}\right)$ is non-negative, ${ }^{n} R_{\alpha}^{x}(x)$ converges. Thus, inequality (2.6) and Lemma 2. 1 show that ${ }^{n} R_{\alpha}^{x}(y)$ converges to an $\alpha$-harmonic function $\widetilde{R}_{\alpha}^{x}(y)$ on $D$ uniformly on any compact subset of $D$, and for each $N$, the restriction of ${ }^{n} R_{a}^{x}$ to $D_{N}$ converges to that of $\widetilde{R}_{a}^{x}$ in the norm $\boldsymbol{D}_{\alpha}^{N}$.

Let us prove that $\widetilde{R}_{\alpha}^{x}(y)=R_{\alpha}^{x}(y), y \in D$. Since $R_{a}^{x}$ belongs to $\boldsymbol{H}_{\alpha}^{n}$, Lemma 2.3 (ii) implies

$$
\boldsymbol{D}_{\alpha}^{n}\left({ }^{n} R_{a}^{x},{ }^{n} R_{a}^{x}\right)-4^{n} R_{a}^{x}(x) \leqq D_{a}^{n}\left(R_{a}^{x}, R_{\alpha}^{x}\right)-4 R_{\alpha}^{x}(x) .
$$

Letting $n$ tend to infinity, we have, for each $N$,

$$
\boldsymbol{D}_{\alpha}^{N}\left(\widetilde{R}_{\alpha}^{x}, \widetilde{R}_{\alpha}^{x}\right)-4 \widetilde{R}_{a}^{x}(x) \leqq \boldsymbol{D}_{a}\left(R_{a}^{x}, R_{a}^{x}\right)-4 R_{a}^{x}(x)
$$

Let $N$ tend to infinity, then

$$
\boldsymbol{D}_{a}\left(\widetilde{R}_{a}^{x}, \widetilde{R}_{a}^{x}\right)-4 \widetilde{R}_{a}^{x}(x) \leqq D_{a}\left(R_{a}^{x}, R_{a}^{x}\right)-4 R_{a}^{x}(x) .
$$

Thus, we see that $\widetilde{R}_{\alpha}^{x} \in \boldsymbol{H}_{\infty}$ and that, by Lemma 2.3 (ii), the inequality above is just the equality and $\tilde{R}_{\alpha}^{x}(y)=R_{\alpha}^{x}(y), y \in D$. The proof of Lemma 2. 5 is complete.

We have seen (in the paragraph following Theorem 1) that, if $\partial D_{n}$ is of class $C^{3},{ }^{n} G_{o}(x, y)$ is nothing but the Laplace transform of the fundamental solution of the heat equation on $D_{n}$ with the boundary condition $\frac{\partial}{\partial n_{x}} u=0$ and this solution is a transition density on $D_{n}$. Hence, we have

Lemma 2.6. Let $\left\{D_{n}\right\},\left\{{ }^{n} R_{\omega}(x, y)\right\}$ and $\left\{{ }^{n}\left(G_{\omega}(x, y)\right\}\right.$ be those in Lemma 2.5. If $D_{n}$ is regular, then we have 


$$
\begin{aligned}
& { }^{n} G_{o}(x, y) \geqq 0, \quad \alpha>0, \quad x, y \in D_{n}, \quad x \neq y . \\
& { }^{n} R_{o}(x, y) \geqq 0, \quad \alpha>0, \quad x, y \in D_{n} . \\
& \alpha \int_{D_{n}}{ }^{n} G_{\infty}(x, y) d y \leqq 1, \quad \alpha>0, \quad x \in D_{n} . \\
& { }^{n} G_{o}(x, y)-{ }^{n} G_{\beta}(x, y)+(\alpha-\beta) \int_{D_{n}}{ }^{n} G_{\infty}(x, z)^{n} G_{\beta}(z, y) d z=0, \\
& \alpha, \beta>0, \quad x, y \in D_{n}, \quad x \neq y .
\end{aligned}
$$

We note that (2. 8) follows from (2. 7).

Now, let us complete the proof of Theorem 1 by the following series of lemmas.

Lemma 2.7. $R_{c o}(x, y)$ is non-negative for $\alpha>0, x, y \in D$ and $\alpha \int_{D} G_{o}(x, y) d y \leqq 1$, for $\alpha>0, x \in D . \quad G_{o s}(x, y)$ is symmetric in $x, y \in D$ and continuous in $(x, y)$ on $D \times D$ off the diagonal.

Proof. The first part of Lemma 2. 7 is an immediate consequence of Lemma 2. 5 and Lemma 2. 6. It is well known that $G_{a}^{0}(x, y)$ is symmetric in $x, y \in D$ and continuous in $(x, y) \in D \times D$ off the diagonal set. $R_{\alpha}(x, y)$ is symmetric because $\boldsymbol{D}_{\alpha}\left(R_{\alpha}^{x}, R_{\alpha}^{y}\right)=2 R_{\alpha}^{x}(y)=2 R_{\alpha}^{y}(x), x, y \in D$.

We shall show that $R_{\omega}(x, y)$ is continuous in $(x, y) \in D \times D$. Since $R_{w}(x, y)$ is $\alpha$-harmonic in $x$ and in $y$, applying Lemma 2. 1 for any $x, y \in D$ and for sufficiently small balls $B_{1}$ and $B_{2}$ containing $x$ and $y$, respectively, we have $R_{\omega}(x, y)=\int_{\partial B_{1}} \int_{\partial B_{2}} h_{\alpha}^{B_{1}}(x, z) R_{\omega}\left(z, z^{\prime}\right) h_{\alpha^{2}}^{B_{2}}\left(y, z^{\prime}\right) \sigma_{1}(d z) \sigma_{2}\left(d z^{\prime}\right)$, where $\sigma_{1}(d z)$ and $\sigma_{2}\left(d z^{\prime}\right)$ are the surface Lebesgue measures of $\partial B_{1}$ and $\partial B_{2}$, respectively. While, $R_{\omega}\left(z, z^{\prime}\right)$ being continuous in $z^{\prime}$ for each $z, \int_{\partial B_{2}} R_{\omega}\left(z, z^{\prime}\right) \sigma_{2}\left(d z^{\prime}\right)$ is finite and $\alpha$-harmonic in $z$. Thus,

$$
\int_{\partial B_{1}} \int_{\partial B_{2}} R_{\infty}\left(z, z^{\prime}\right) \sigma_{1}(d z) \sigma_{2}\left(d z^{\prime}\right)<+\infty .
$$

Since $R_{a}$ is non-negative, Lebesgue's convergence theorem implies continuity of $R_{\infty}(x, y)$. The proof of the latter half of Lemma 2. 7 is complete.

We will show assertion (iv) of Theorem 1 .

Lemma 2.8. Let $K_{1}$ and $K_{2}$ be compact subsets of $D$ such that $K_{1}$ and the closure of $D-K_{2}$ are disjoint. Then, $\sup _{x \in K_{1}, y \in D-K_{2}} G_{\infty}(x, y)$ is finite.

Proof. Without loss of generality, we can assume that $S=\partial\left(D-K_{2}\right) \cap D$ is sufficiently regular. Consider a regular exhaustion $\left\{D_{n}\right\}$ of $D$ such that $D_{1} \supset K_{2}$. Let $x$ be fixed in $K_{1}$. For a fixed $n$, set $D^{\prime}=D_{n}-K_{2}$ and $u(y)$ 
$={ }^{n} G_{\omega}(x, y), y \in D^{\prime} \cup \partial D^{\prime}$. Since $\frac{\partial}{\partial n_{y}} u(y)=0, y \in \partial D_{n}$, we see by Green's formula that $\boldsymbol{D}_{a}{ }^{\prime}(u, v-u)=0$ holds if $v \in \boldsymbol{C}^{1}\left(D^{\prime} \cup \partial D^{\prime}\right)$ and $v=u$ on $S^{8)}$. Hence, the equality

$$
\boldsymbol{D}_{a s}{ }^{\prime}(u, u)=\boldsymbol{D}_{a s}{ }^{\prime}(v, v)-\boldsymbol{D}_{a s}{ }^{\prime}(u-v, u-v)
$$

is valid for each $v$ belonging to $\mathfrak{D}_{u}=\left\{v ; v\right.$ is square summable on $D^{\prime}, v$ has square summable weak-derivatives on $D^{\prime}, v \in C\left(D^{\prime} \cup S\right)$ and $v=u$ on $\left.S\right\}^{9)}$. Set $\delta=\sup _{y \in S} u(y)$ and $u_{1}(y)=\min (u(y), \delta), y \in D^{\prime} \cup S$. Obviously, $\boldsymbol{D}_{a}{ }^{\prime}(u, u)$ $\geqq \boldsymbol{D}_{a}{ }^{\prime}\left(u_{1}, u_{1}\right)$. But, since $u_{1} \in \mathscr{D}_{u},(2.11)$ holds for $v=u_{1}$ and consequently $u_{1}(y)=u(y)$ on $D^{\prime}$.

We have proved that, if $x \in K_{1}$ and $y \in D_{n}-K_{2}$, then ${ }^{n} G_{a}(x, y)$ $\leqq \sup _{y \in S}{ }^{n} G_{w}(x, y)$. Letting $n$ tend to infinity, we see by virtue of Lemma 2. 5, $G_{o}(x, y) \leqq \sup _{y \in S} G_{o}(x, y), x \in K_{1}, y \in D-K_{2}$. Thus,

$$
\sup _{x \in K_{1}, y \in D-\kappa_{2}} G_{a s}(x, y) \leqq \sup _{x \in K_{1}, y \in S} G_{a b}(x, y) .
$$

The right hand side above is finite by Lemma 2.7 .

Let us show statement (iii) of Theorem 1.

Lemma 2.9. The operator $G_{a}$ defined by (1.6) maps $\boldsymbol{B}(D)$ into $\boldsymbol{C}(D)$. Moreover, if $f \in C(D)$, then $\lim _{\alpha \rightarrow+\infty} \alpha G_{a} f(x)=f(x), x \in D$.

Proof. We note that $G_{\alpha}^{0}$ has those properties in Lemma 2. $9^{10)}$. For $f \in \boldsymbol{B}(D), R_{\alpha} f(x)=\int_{D} R_{\omega}(x, y) f(y) d y$ is $\alpha$-harmonic and bounded on account of Lemma 2. 1 and Lemma 2. 7. Moreover, we see by Lemma 2. 1 that, for any $x \in D$ and sufficiently small ball $B$ containing $x$.,

$$
\begin{aligned}
& \left|\alpha R_{\alpha} f(x)\right| \leqq \int_{\partial B} h_{\alpha}^{B}(x, y)\left|\alpha R_{\alpha} f(y)\right| \sigma(d y) \\
& \leqq \sup _{x \in D}|f(x)| \int_{\partial B} h_{\alpha}^{B}(x, y) \sigma(d y) \underset{\alpha \rightarrow+\infty}{\longrightarrow} 0 .
\end{aligned}
$$

The proof of Lemma 2. 9 is complete.

The following lemmas are statements (ii) and (v) of Theorem 1.

8) $\boldsymbol{D}_{\alpha}{ }^{\prime}$ denotes the integral (2.1) on $D^{\prime}$.

9) We call $f$ the weak derivative of $v$ with respect to the coodinate $x_{i}$, if $(f, \varphi)_{D^{\prime}}$ $=-\left(v, \frac{\partial}{\partial x_{i}} \varphi\right)_{D^{\prime}}$ holds for every infinitely differentiable function on $D^{\prime}$ with a compact support, $(,)_{D^{\prime}}$ being the integral (1.3) on $D^{\prime}$.

10) See (1. 2). 
Lemma 2.10. $G_{o b}(x, y)$ is a conservative resolvent density on $D . \quad R_{o b}(x, y)$ is strictly positive.

Proof. We must prove that $G_{a}(x, y)$ satisfies conditions (G. 1) (G. 4) stated in the beginning of Section 1 and the conservativity condition. Condition (G. 1), (G. 2) and (G. 4) were already proved in Lemma 2. 7.

Proof of the resolvent equation (G. 3). Take a regular exhaustion $\left\{D_{n}\right\}$ of $D$. Let $f$ and $g$ be non-negative continuous functions on $D$ with compact supports. Owing to equation (2.10) of Lemma 2. 6, we have for sufficiently large $n$,

$$
\left(f,{ }^{n} G_{a} g\right)_{n}-\left(f,{ }^{n} G_{\beta} g\right)_{n}+(\alpha-\beta)\left({ }^{n} G_{o s} f,{ }^{n} G_{\beta} g\right)_{n}=0,
$$

where $(u, v)_{n}$ denotes the integral of $u v$ on $D_{n}$.

Note that $0 \leqq{ }^{n} G_{o} f(x){ }^{n} G_{\beta} g(x) \leqq \frac{1}{\alpha \beta} \sup _{x \in D} f(x) \cdot \sup _{x \in D} g(x)$ and that ${ }^{n} G_{\infty} g$ converges to $G_{a} g$ on $D$ (since, ${ }^{n} G_{\alpha}^{0} g$ increases to $G_{\alpha}^{0} g$ and ${ }^{n} R_{\alpha}^{x}(y)$ converges uniformly on any compact subset).

Hence, we can delete both superscript and subscript $n$ in (2. 12). Owing to Lemma 2. 8 and Lemma 2. 9, the left hand side of (G. 3) is, for each $x \in D$, continuous in $y \in D-\{x\}$, and we can see that the resolvent equation (G. 3 ) is valid.

Proof of conservativity. If we show that $R_{a} 1 \in \boldsymbol{H}_{a}$ and that

$$
\boldsymbol{D}_{\alpha}\left(\alpha R_{o} 1, v\right)=2 \alpha(1, v),
$$

holds for all $v \in \boldsymbol{H}_{\alpha}$, then, we have, by (ii) of Lemma 2. 4, $1-\alpha G_{\alpha}^{0} 1=\alpha R_{a} 1$ and $\alpha G_{o 1} 1=1$.

Let $D_{n}$ be an exhaustion of $D$. Integrating $\boldsymbol{D}_{a}\left(R_{\alpha}^{x}, R_{\alpha}^{y}\right)=2 R_{a}(x, y)$ on $D_{m} \times D_{n}$, we obtain

$$
\boldsymbol{D}_{a s}\left(R_{o s} \chi_{D_{m}}, R_{a s} \chi_{D_{n}}\right)=2 \int_{D_{m}} \int_{D_{n}} R_{\omega s}(x, y) d x d y .
$$

Here, we have used the Fubini theorem, which is valid for the following reason: if $m \leqq n$,

$$
\begin{aligned}
& \int_{D_{m}} \int_{D_{n}} d x d y \int_{D}\left|\left(\operatorname{grad}_{z} R_{\alpha}^{x}(z), \operatorname{grad}_{z} R_{\alpha}^{y}(z)\right)\right| d z \\
& \leqq \int_{D_{n}} \int_{D_{n}} \sqrt{D_{o s}\left(R_{\alpha}^{x}, R_{a}^{x}\right)} \sqrt{ } \overline{D_{a}\left(R_{\alpha}^{y}, R_{a}^{y}\right)} d x d y \\
& =\left(\int_{D_{n}} \sqrt{2 R_{\infty}(x, x)} d x\right)^{2} \leqq 2 \int_{D_{n}} R_{\omega s}(x, x) d x \times \text { Lebesgue measure of } D_{n} \text {, }
\end{aligned}
$$

the integral in the last expression being finite by Lemma 2.7. In view of 
Lemma 2. 7, $R_{\infty}(x, y) \geqq 0$ and $\int_{D} \int_{D} R_{\infty}(x, y) d x d y \leqq \frac{1}{\alpha} \times$ Lebesgue measure of $D$. Therefore, $R_{a} \chi_{D_{n}}$ forms a Cauchy sequence in $\boldsymbol{H}_{a}$ and, by Lemma 2.2, converges to $R_{a b} 1$ in $\boldsymbol{H}_{a}$. We have $\boldsymbol{D}_{a}\left(R_{a} 1, R_{a} 1\right)=2\left(1, R_{a} 1\right)$. In the same way, identity (2.13) is obtained. Strict positivity of $R_{\omega}(x, y)$ follows from Lemma 2. 4.

Lemma 2.11. There is a unique transition density $p(t, x, y)$ on $D$ satisfying the following conditions.

(i ) $G_{\omega}(x, y)=\int_{0}^{+\infty} e^{-\infty t} p(t, x, y) d t, \quad \alpha>0$.

(ii) For each $\boldsymbol{t}>0, f \in \boldsymbol{B}(D)$,

$$
\int_{D} p(t, x, y) f(y) d y \text { is continuous in }(t, x) \in(0,+\infty) \times D .
$$

(iii) $p(t, x, y)$ is symmetric in $x, y \in D$ and it is conservative.

(iv) Set $\gamma(t, x, y)=p(t, x, y)-p^{0}(t, x, y)$, then

$$
\frac{1}{t} \int_{D} \gamma(t, x, y) d y \underset{t \rightarrow 0}{\longrightarrow} 0 \text { uniformly in } x \text { on any compact subset of } D \text {. }
$$

Proof. First of all, we will show the existence of a non-negative function $\gamma(t, x, y)$ continuous in $t>0$, satisfying

$$
R_{\alpha}(x, y)=\int_{0}^{+\infty} e^{-\alpha t} \gamma(t, x, y) d t, \quad \alpha>0, \quad x, y \in D .
$$

If $x \neq y, R_{a}(x, y)$ is completely monotonic in $\alpha \in(0,+\infty)$. In fact, by the resolvent equation (G. 3) for $G_{a}$ and $G_{\alpha}^{0}$, we have, if $x \neq y$,

$$
(-1)^{n} \frac{d^{n}}{d \alpha^{n}} R_{\alpha s}(x, y)=n !\left[G_{\alpha}^{[n+1]}(x, y)-\left(G_{\alpha}^{0}\right)^{[n+1]}(x, y)\right], \quad n=0,1,2, \cdots
$$

Here $G_{\alpha}^{[1]}(x, y)=G_{o x}(x, y)$ and $G_{\alpha}^{[n+1]}(x, y)=\int_{D} G_{\alpha}^{[n]}(x, z) G_{o s}(z, y) d z, n=1,2, \cdots$. $\left(G_{a}^{0}\right)^{[n]}$ is defined similarly. Evidently, the right hand side of (2.16) is nonnegative and, by Lemma 2.8 , finite. Hence, $R_{b}(x, y)$ is expressed by a measure on $[0,+\infty)$ as

$$
R_{a s}(x, y)=\int_{0}^{+\infty} e^{-a s} \gamma(d s, x, y), \quad x \neq y, \quad \alpha>0
$$

Take a ball $B$ with closure contained in $D$. Since $R_{a}(x, y)$ is $\alpha$-harmonic in $x$, we see, by Lemma 2.1 , for any $x \in B$ and any $y \in D$,

$$
R_{a}(x, y)=\int_{\partial B} h_{a}^{B}(x, z) R_{o s}(z, y) \sigma(d z)
$$


Note that $h_{a}^{B}(x, z)$ is written in the form

$$
h_{\alpha}^{B}(x, z)=\int_{0}^{+\infty} e^{-\alpha t} h^{B}(t, x, z) d t, \quad z \in B, \quad z \in \partial B,
$$

where $h^{B}(t, x, z)=\frac{1}{2} \frac{\partial}{\partial n_{z}} p_{B}^{0}(t, x, z), p_{B}^{0}$ being the transition density $p^{0}$ for $B$. Let us put, for $t>0, x \in B$ and $y \in D$,

$$
\gamma(t, x, y)=\int_{\partial B} \int_{0}^{t} h^{B}(t-s, x, z) \gamma(d s, z, y) \sigma(d z) .
$$

Owing to equations (2.17), (2.18) and (2.19), the function $\gamma(t, x, y)$ of (2. 20) satisfies the desired equation (2.15). On the other hand, for any ball $B^{\prime}$ such as $B^{\prime} \cup \partial B^{\prime} \subset B$, the obvious idenity $h^{B}(t, x, z)=\int_{\partial B^{\prime}} \int_{0}^{t} h^{B^{\prime}}\left(t-s, x, z^{\prime}\right) h^{B}\left(s, z^{\prime}, z\right)$ $d s \sigma^{\prime}\left(d z^{\prime}\right), x \in B^{\prime}, z \in \partial B$,

leads us to the relation

$$
\begin{aligned}
& \gamma(t, x, y)=\int_{\partial B^{\prime}} \int_{0}^{t} h^{B^{\prime}}\left(t-s, x, z^{\prime}\right) \gamma\left(s, z^{\prime}, y\right) d s \sigma^{\prime}\left(d z^{\prime}\right), \\
& t>0, \quad x \in B^{\prime} \quad y \in D
\end{aligned}
$$

which implies the continuity of $\gamma(t, x, y)$ in $(t, x) \in(0,+\infty) \times B^{\prime}$.

Here, we have used the following estimate which is a consequence of (2. 17), (2. 20) and Lemma 2. 8.

$$
\sup _{0<t \leqq T, x \in B^{\prime}, v \in D} \gamma(t, x, y) \leqq C \cdot e^{T} \cdot \sup _{z \in \partial B, y \in D} R_{1}(z, y)<+\infty,
$$

where $T$ is an arbitrary positive number and $C$ is a constant determined by $T$, $B$ and $B^{\prime}$. Hence, we see that, for any $x$ and $y$ in $D, \gamma(t, x, y)$ defined by (2. 20) is independent of ball $B$ such that $x \in B$ and $B \cup \partial B \subset D$, because it satisfies (2.15) and it is continuous in $t$. It is symmetric in $x, y$ because of the symmetry of $R_{a x}(x, y)$ (Lemma 2.7). Henceforce, it is continuous in $y$, and (2. 21) and (2. 22) imply its continuity in $(t, x, y) \in(0,+\infty) \times D \times D$. In view of (2. 22), we see that $\int_{D} \gamma(t, x, y) f(y) d y$ is continuous in $(t, x) \in(0,+\infty) \times D$ for each $f \in \boldsymbol{B}(D)$.

Now put, for $t>0, x, y \in D$,

$$
p(t, x, y)=p^{0}(t, x, y)+\gamma(t, x, y) \text {. }
$$

Then, $p(t, x, y)$ is continuous in $(t, x, y) \in(0,+\infty) \times D \times D$ and satisfies conditions (i), (ii) and the first half of Lemma 2. 11 (iii). In particular, $\int_{D} p(t, x, y) d y$ is continuous in $t$, so that, the conservativity of $p(t, x, y)$ follows 
from that of $G_{\omega}(x, y)$. For each $x, y \in D, p(t+s, x, y)$ and $\int_{D} p(t, x, z)$ $p(s, z, y) d z$ are continuous in $(t, s) \in(0,+\infty) \times(0,+\infty)$, and so, they are identical by virtue of (G. 3$)$ for $G_{o x}(x, y)$. Thus, $p(t, x, y)$ is a transition density. Assersion (iv) of Lemma 2.11 follows from (2.21) and the inequality $\int_{D} \gamma(t, x, y) d y \leqq 1, t>0, x \in D$.

\section{Compactification of D. Construction of a strong Markov process on the compactified space}

Consider the resolvent density $G_{a}(x, y), \alpha>0, x, y \in D$, in Theorem 1 . Let $x_{n} \in D, n=1,2, \cdots$, be a sequence having no accumulation point in $D$ and $\left\{D_{l}, l=1,2, \cdots\right\}$ be an exhaustion of $D$. For each $l$, there exists $N$ such that $x_{n} \in D-D_{l+2}, n \geqq N$. By Theorem 1 (iv), the family of functions $\left\{G_{1}\left(x_{n}, y\right)\right.$, $n \geqq N\}$ of $y$ is uniformly bounded in $y \in D_{l+1}$. Moreover, Lemma 2.1 implies that, for $n \geqq N$, the first derivatives of $G_{1}\left(x_{n}, y\right), n \geqq N$, are also uniformly bounded in $y \in D_{l}$ and that functions $G_{1}\left(x_{n}, y\right), n \geqq N$, are equi-continuous there. Hence, a subsequence of $G_{1}\left(x_{n}, y\right)$ converges uniformly on each $D_{l}$ and consequently, by Lemma 2.1 , the limit function is 1-harmonic in $D$.

A sequence $x_{n} \in D, n=1,2, \cdots$ having no accumulation point in $D$ is called fundamental, if $\lim _{n \rightarrow+\infty} G_{1}\left(x_{n}, y\right)$ exists for each $y \in D$.

Two fundamental sequences $\left\{x_{n}\right\}$ and $\left\{x_{n}{ }^{\prime}\right\}$ are called equivalent, if $\lim _{n \rightarrow+\infty} G_{1}\left(x_{n}, y\right)=\lim _{n \rightarrow+\infty} G_{1}\left(x_{n}{ }^{\prime}, y\right), y \in D$. This defines a usual equivalence relation among fundamental sequences.

\section{Definition 3.}

(i) Denote by $\triangle$ the collection of equivalent classes of fundamental sequences.

(ii) For $x \in \triangle$, define $G_{1}(x, y)$ by $G_{1}(x, y)=\lim _{n \rightarrow+\infty} G_{1}\left(x_{n}, y\right), y \in D$, where, $\left\{x_{n}\right\}$ is a fundamental sequence belonging to $x$.

(iii) Set $D^{*}=D \cup \triangle$. For $x_{1}, x_{2} \in D^{*}$, set

$$
\rho\left(x_{1}, x_{2}\right)=\int_{D} \frac{\left|G_{1}\left(x_{1}, y\right)-G_{1}\left(x_{2}, y\right)\right|}{1+\left|G_{1}\left(x_{1}, y\right)-G_{1}\left(x_{2}, y\right)\right|} d y .
$$

Evidently, $\rho$ defines a metric on $D^{*}$.

\section{Lemma 3.1.}

(i) $\left(D^{*}, \rho\right)$ is a compactification of $D$.

(ii) For each $y$ in $D$, the extended function $G_{1}(x, y)$ is $\rho$-continuous in $x$ on $D^{*}-\{y\}$ and the class of functions (of $\left.x\right),\left\{G_{1}(x, y), y \in D\right\}$, separates points of $D^{*}$. 
(iii) If $K$ is a compact subset of $D$ and $F$ is a closed subset of $D^{*}-K$, then $\sup _{x \in F, y \in K} G_{1}(x, y)$ is finite.

(iv) When the relative boundary $\partial D$ of $D$ in $R^{N}$ is of class $C^{3}, D \cup \partial D$ coincides with $D^{*}$ up to a homeomorphism which is the identity on $D$.

Proof. Martin's original proof (cf. [13], §2, Theorem I and II) can be applied with no change to obtain the statements (i) and (ii). Third assertion is a consequence of Theorem 1 (iv). Suppose that $\partial D$ is of class $\boldsymbol{C}^{3}$. As we have seen in Section $1, G_{\infty}(x, y)$ of Theorem 1 is the Laplace transform of a fundamental solution $\dot{p}(t, x, y)$ of a boundary problem of the heat equation. $\dot{p}(t, x, y)$ and $G_{o s}(x, y)$ can be continuously extended to $D \cup \partial D$ as functions of $x$ and it holds that, for each $x \in D \cup \partial D, f \in C(D \cup \partial D), \lim _{t \rightarrow 0} \int_{D} \dot{p}(t, x, y)$ $f(y) d y=f(x)^{11)}, \quad$ which implies $\lim _{\alpha \rightarrow+\infty} \alpha \int_{D} G_{o}(x, y) f(y) d y=f(x)$. Hence, $\left\{G_{1}(x, y), y \in D\right\}$ separate points of $D \cup \partial D$. Therefore, $D \cup \partial D$ is homeomorphic to $D^{*}$ (cf. [1], §9).

Denote by $\mathfrak{B}\left(D^{*}\right)$ the $\sigma$-field of all Borel subsets of $D^{*} . \boldsymbol{B}\left(D^{*}\right), \boldsymbol{C}\left(D^{*}\right)$ and $C_{0}(D)$ will stand for the classes of all bounded Borel measurable functions on $D^{*}, \rho$-continuous functions on $D^{*}$ and continuous functions on $D$ with compact supports in $D$, respectively. Each $f \in C_{0}(D)$ will be considered as a function on $D^{*}$ by setting $f(x)=0, x \in \triangle$.

As an immediate consequence of Lemma 3. 1 and Theorem 1 (iii), we have

Corollary. The operator $G_{1}$, defined by $G_{1} f(x)=\int_{D} G_{1}(x, y) f(y) d y, x \in D^{*}$, maps $C_{0}(D)$ into $C\left(D^{*}\right)$ and the collection of functions $G_{1} f, f \in C_{0}(D)$, separates points of $D^{*}$.

Now, let us extend every function $G_{w}(x, y), \alpha>0$, as follows.

Definition 4. For $\alpha>0, x \in \triangle, y \in D$, define $G_{w}(x, y)$ by

$$
G_{\omega}(x, y)=G_{1}(x, y)-(\alpha-1) \int_{D} G_{1}(x, z) G_{o x}(z, y) d z .
$$

Lemma 3.2. For each $x \in \triangle, G_{w}(x, y)$ has the following properties:

(G. 1)' $G_{a}(x, y), \alpha>0, y \in D$, is non-negative, finite and $\alpha$-harmonic in $y \in D$,

(G. 2) $\quad \alpha G_{\omega} 1(x)=G_{1} 1(x) \leqq 1, \quad \alpha>0$,

where $G_{\infty} 1(x)=\int_{D} G_{a}(x, y) d y$.

$(\mathrm{G} .3)^{\prime} \quad G_{\alpha}(x, y)-G_{\beta}(x, y)+(\alpha-\beta) \int_{D} G_{\omega}(x, z) G_{\beta}(z, y) d z=0, \alpha, \beta>0, y \in D$.

11) cf. [7]. 
Proof. Let us fix $x \in \triangle$. By Fatou's lemma,

$$
G_{1} 1(x) \leqq 1
$$

By virtue of (3.3), assertion (iii) of Lemma 3. 1 and assertion (iv) of Theorem 1 , the integral appering in (3.2) turns out to be finite for $\alpha>0$ and $y \in D$. When $\alpha<1, G_{a}(x, y)$ is clearly non-negative. By Fatou's lemma, $G_{a}(x, y) \geqq 0$ for $\alpha>1$. We can easily verify

$$
\left(\alpha-\frac{1}{2} \triangle_{y}\right) G_{o}(x, y)=0, \quad \alpha>0, \quad y \in D .
$$

Integrating both sides of (3.2) in $y$ and noting the conservativity of $G_{a}$ of Theorem 1, we get $\alpha G_{a} 1(x)=G_{1} 1(x), \alpha>0$. The equation (G. 3)' is obtained from (3. 2) by a simple calculation.

We now extend $p(t, x, y)$ of Thoerem 1 (v) from $D$ to $D^{*}$ with respect to $x$.

Lemma 3.3. For each $x \in \triangle$, there is one and only one function $p(t, x, y)$, $t>0, y \in D$, which is continuous in $t$ and satisfies

$$
G_{\omega}(x, y)=\int_{0}^{+\infty} e^{-\alpha t} p(t, x, y) d t, \quad \alpha>0, \quad y \in D .
$$

Moreover the function $p(t, x, y)$ has the following properties:

(T. 1)' It is non negative.

(T. 2)' $\int_{D} p(t, x, y) d y=G_{1} 1(x) \leqq 1, \quad t>0$.

(T. 3)' $\int_{D} p(t, x, z) p(s, z, y) d z=p(t+s, x, y), \quad t, s>0, \quad y \in D$.

$(\mathrm{T} .4)^{\prime}$ For each $x \in \triangle$, it is continuous in $(t, y) \in(0,+\infty) \times D$ and, for each $t>0$ and $y \in D$, it is measurable in $x$ on $\triangle$. Moreover, for any $f \in \boldsymbol{B}\left(D^{*}\right)$ and $x \in \triangle, \int_{D} p(t, x, y) f(y) d y$ is continuous in $t>0$.

Proof. In view of (G. 3)' of Lemma 3. 2, we see that $G_{x}(x, y), x \in \triangle$, $y \in D$ is completely monotonic in $\alpha \in(0,+\infty)$. By (G. 1)' of Lemma 3. 2, it is $\alpha$-harmonic in $y \in D$. Hence, we can construct $p(t, x, y), t>0, x \in \triangle, y \in D$, satisfying (3. 4), (T. 1)' and the first half of (T. 4)' in the same manner as the construction of $\gamma(t, x, y)$ of Lemma 2. 11 .

As consequences of properties (G. 2)' and (G. 3)' of Lemma 3. 2, the equation in ('T. 2)' holds for almost all $t>0$ and relation (T. 3) ${ }^{\prime}$ holds for almost all $t, s>0$. By virtue of (2. 22), the left hand side of (T. 3)' is continuous in $s>0$ for each $t$ satisfying (T. 2) $)^{\prime}$. So the equation (T. 3)' holds for almost all $t>0$ and for all $s>0$. In view of property (T. 3 ) of the transition density $p(t, x, y)$, 
$t>0, x, y \in D$, (T. 3) $)^{\prime}$ holds for all $t, s>0$. (T. 3$)^{\prime}$ implies that the left hand side of (T. 2)' is a constant in $t$. Hence (T. 2)' holds for all $t>0$. It follow from the first half of (T. 4$)^{\prime}$ that $\int_{D} p(t, x, y) f(y) d y$ is lower semi-continuous in $t$ for each non-negative bounded function $f$ on $D$. Moreover, on account of (T. 2)', it is continuous in $t$. Thus, $\int_{D} p(t, x, y) f(y) d y$ is continuous in $t>0$ for each $f \in \boldsymbol{B}\left(D^{*}\right)$ and $x \in \triangle$.

Now, we are in a position to construct the Markov process (on $D^{*}$ ) associated with $p(t, x, y), x \in D^{*}, y \in D$, and investigate its properties.

Add a point $\partial$ to $D^{*}$ as an isolated point. $\mathfrak{B}\left(D^{*} \cup \partial\right)$ will stand for the collection of sets whose restrictions to $D^{*}$ are the elements of $\mathfrak{B}\left(D^{*}\right)$. Denote by $\boldsymbol{B}\left(D^{*} \cup \partial\right)\left(\boldsymbol{C}\left(D^{*} \cup \partial\right)\right)$ the aggregate of all the functions on $D^{*} \cup \partial$ whose restrictions to $D^{*}$ are the elements of $\boldsymbol{B}\left(D^{*}\right)$ (resp. $\boldsymbol{C}\left(D^{*}\right)$ ). Each element $f$ of $\boldsymbol{B}\left(D^{*}\right)$ will always be considered as the one of $\boldsymbol{B}\left(D^{*} \cup \partial\right)$ by setting $f(\partial)=0$, unless particularly mentioned. Let $p(t, x, y)$ be the function defined for $t>0$, $x \in D^{*}$ and $y \in D$ by Theorem 1 (v) and Lemma 3.3. For $E \in \boldsymbol{B}\left(D^{*} \cup \partial\right)$, define

$$
\begin{aligned}
& p(t, x, E)=\int_{E \cup D} p(t, x, y) d y+(1-q(x)) \chi_{E}(\partial), \quad x \in D^{*}, \\
& p(t, \partial, E)=\chi_{E}(\partial),
\end{aligned}
$$

where $\chi_{E}$ is the indicator function of the set $E$, and

$$
q(x)=\int_{D} G_{1}(x, y) d y, \quad x \in D^{*} .
$$

We put for $f \in \boldsymbol{B}\left(D^{*} \cup \partial\right)$,

$$
\begin{aligned}
T_{t} f(x) & =\int_{D^{*} \cup \partial} p(t, x, d y) f(y), \\
G_{a} f(x) & =\int_{0}^{+\infty} e^{-\alpha t} T_{t} f(x) d t, \quad x \in D^{*} \cup \partial, \quad t>0, \quad \alpha>0 .
\end{aligned}
$$

$G_{a} f$ is expressed in the form

$$
\begin{aligned}
G_{a} f(x) & =\int_{D} G_{a}(x, y) f(y) d y+\frac{1-q(x)}{\alpha} f(\partial), \quad x \in D^{*}, \\
G_{a} f(\partial) & =\frac{f(\partial)}{\alpha} .
\end{aligned}
$$

By virtue of Theorem $1(\mathrm{v})$ and Lemma 3. 3, $p(t, x, E)$ defined by (3. 5) is a transition function on $D^{*} \cup \partial ; p(t, x, \cdot)$ is a probability measure on $D^{*} \cup \partial$, $p(\cdot, \cdot, E)$ is, for each $E \in \mathfrak{B}\left(D^{*} \cup \partial\right)$, measureable in $(t, x) \in(0,+\infty) \times\left\{D^{*} \cup \partial\right\}$ 
and it satisfies the Chapmann-Kolmogorov equation.

Let $\Omega$ be the product compact space $\left\{D^{*} \cup \partial\right\}^{(0,+\infty)}$. Denote by $\tilde{X}_{t}(\omega)$ the $t$-th coodinate of $\omega \in \Omega$. Let $\mathfrak{F}\left(\mathfrak{F}_{t}\right)$ be the $\sigma$-field of subsets of $\Omega$ generated by the cylindrical open sets of $\Omega$ (resp. cylindrical open sets depending on the coodinates up to and including $t$ ). Denote by $\mathfrak{A}$ the $\sigma$-field of subsets of $\Omega$ generated by all open set of $\Omega$. For each $x \in D^{*} \cup \partial$, there is a unique Radon measure ${ }^{12)} P_{x}$ over $(\Omega, \mathfrak{U})$ which is a probability measure and satisfies the following conditions.

$$
\begin{aligned}
& P_{x}\left(\tilde{X}_{t} \in E\right)=p(t, x, E), \\
& t>0, \quad x \in D^{*} \cup \partial, \quad E \in \mathfrak{B}\left(D^{*} \cup \partial\right),
\end{aligned}
$$

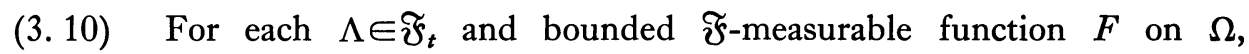
$E_{x}\left(F_{x}\left(\theta_{t} \omega\right) ; \Lambda\right)=E_{x}\left(E_{\tilde{X}_{t}}(F) ; \Lambda\right), x \in D^{*} \cup \partial$, where $E_{x}$ denotes the integration with respect to $P_{x}$-measure and $\theta_{t} ; t>0$, is the shift from $\Omega$ to $\Omega$ defined by $\tilde{X}_{s}\left(\theta_{t} \omega\right)=\tilde{X}_{t+s}(\omega), s>0$.

\section{Lemma 3.4.}

(i) Set $\Omega_{1}=\left\{\omega ; \tilde{X}_{t}(\omega) \in D^{*}\right.$ for every $\left.t>0\right\}$ and $\Omega_{2}=\left\{\omega: \tilde{X}_{t}(\omega) \in\{\partial\}\right.$ for every $t>0\}$. Then, $P_{x}\left(\Omega_{1}\right)=q(x), P_{x}\left(\Omega_{2}\right)=1-q(x), x \in D^{*}$ and $P_{\{\partial\}}\left(\Omega_{2}\right)=1$.

(ii) For each $x \in D^{*} \cup \partial$, we have $P_{x}\left(\tilde{X}_{t}\right.$ has the right limits for all $t \geqq 0$ and the left limits for all $t>0)=1$.

Proof. (i). Relations (3. 5), (3. 9) and (3. 10) imply $P_{x}\left(\tilde{X}_{t} \in D^{*}\right.$, $\left.\tilde{X}_{s} \in\{\partial\}\right)=0$ for every $t, s$ such as $t>s>0$ and for every $x \in D^{*}$. Since $\left\{\tilde{X}_{t}, P_{x}\right\}, x \in D^{*}$, is separable, ${ }^{13)}$ we see $P_{x}\left(\Omega_{1}\right)=\lim _{t \rightarrow+\infty} P_{x}\left(\tilde{X}_{t} \in D^{*}\right)=q(x)$ and $P_{x}\left(\Omega_{2}\right)=\lim _{t \rightarrow 0} P_{x}\left(\tilde{X}_{t} \in\{\partial\}\right)=1-q(x)$.

(ii). Denote by $\boldsymbol{C}_{0}^{+}(D)$ the collection of all non-negative functions in $\boldsymbol{C}_{0}(D)$ and by $\boldsymbol{S}_{0}(D)$ a countable dense subset of $\boldsymbol{C}_{0}^{+}(D)$ in uniform norm. By virtue of Corollary to Lemma 3. 1, functions $G_{1} f, f \in \boldsymbol{S}_{0}(D)$, are continuous on $D^{*}$ and separate points of $D^{*}$. Moreover, $\left\{Z_{t}=e^{-t} G_{1} f\left(\tilde{X}_{t}\right), \mathfrak{F}_{t}, P_{x}\right\}, f \in \boldsymbol{S}_{0}(D)$, $x \in D^{*}$, is a bounded supermartingale. Hence, we have assertion (ii) by a standard argument ${ }^{14)}$.

It follows from Lemma 3. 5 that there is well defined $X_{t}(\omega)=\lim _{t^{\prime} \downarrow t} \tilde{X}_{t^{\prime}}(\omega)$ for every $t \geqq 0$ almost everywhere $\left(P_{x}\right), x \in D^{*} \cup \partial . \quad X_{t}$ is right continuous in $t \geqq 0$ and has the left limit in $t>0$ almost everywhere $\left(P_{x}\right), x \in D^{*} \cup \partial$. On account of Theorem $1(\mathrm{v})$ and Lemma $3.3(\mathrm{~T} .4)^{\prime}, X_{t}$ is a modification of $\tilde{X}_{t}$; $P_{x}\left(X_{t}=\tilde{X}_{t}\right)=1$, for each $t>0$ and $x \in D^{*} \cup \partial$.
12) cf. [15].
13) cf. [15].
14) cf. $[10]$ and $[20]$. 
Let us examine the distribution of $X_{0}$.

\section{Definition 5.}

(i) For each $x \in D^{*} \cup \partial$, define a probability measure $\mu(x, E)$ on $\mathfrak{B}\left(D^{*} \cup \partial\right)$ by

$$
\mu(x, E)=P_{x}\left(X_{0} \in E\right), \quad E \in \mathfrak{B}\left(D^{*} \cup \partial\right) .
$$

This $\mu(x, \cdot)$ is called the branching measure at $x$.

(ii) A point $x$ in $D^{*} \cup \partial$ is called a branching point if $\mu(x,\{x\})<1$.

The notion of branching measure was introduced by $D$. Ray [20]. The above definition, slightly different from Ray's original one, is due to H. Kunita and $\mathrm{T}$. Watanabe [10]. We shall use the general results obtained by these authors, whenever their methods of the proof are applicable to our situation without essential change.

Denote by $\triangle_{0}$ the totality of branching points. Then, we have

\section{Lemma 3.5.}

(i) $\triangle_{0} \subset \triangle$.

(ii) $\triangle_{0}$ is an $F_{\sigma}$ set and $\mu\left(x, \triangle_{0}\right)=0, x \in \triangle_{0}$.

(iii) Put $\triangle_{0}^{\prime}=\{x: q(x)<1\}$, where $q(x)=\int_{D} G_{1}(x, y) d y$. Then, $\triangle_{0}{ }^{\prime} \subset \triangle_{0}$ and $\mu(x,\{\partial\})=1-q(x), x \in \triangle_{0}$.

Proof. If $f \in \boldsymbol{C}\left(D^{*} \cup \partial\right)$, then

$$
\begin{aligned}
& \lim _{\alpha \rightarrow+\infty} \alpha G_{a x} f(x)=\lim _{\alpha \rightarrow+\infty} E_{x}\left(\int_{0}^{+\infty} e^{-t} f\left(X_{t / \alpha}\right) d t\right) \\
& =E_{x}\left(f\left(X_{0}\right)\right)=\int_{D^{*} \cup \partial} \mu(x, d y) f(y), \quad x \in D^{*} \cup \partial .
\end{aligned}
$$

On the other hand, because of Theorem 1 (ii) and formula (3. 8), $\lim _{\alpha \rightarrow+\infty} \alpha G_{a} f(x)$ $=f(x)$, for $x \in D \cup \partial, f \in C\left(D^{*} \cup \partial\right)$. Hence, $D \cup \partial$ contains no branching point.

For the proof of (ii), let us cite a criterion of D. Ray [20] in a modified form fitted to our situation: $x \in \triangle_{0}$, if and only if $f(x)>\lim _{\alpha \rightarrow+\infty} \alpha G_{a f} f(x)$, for some $f \in \boldsymbol{C}_{1}=\left\{f=G_{1} h \wedge c ; h \in_{0} \mathbf{S}(D), c\right.$ is non-negative rational $\}$. Since, for $f \in \boldsymbol{C}_{1}$, $\alpha G_{a+1} f \leqq f$ and $G_{a+1} f=G_{1}\left(f-\alpha G_{\omega+1} f\right)$ is lower semi-continuous on $D^{*}$, $\triangle_{0}=\bigcup_{f \in C_{1}} \bigcup_{n=1}^{+\infty} \bigcap_{\alpha>0, \text { rational }}\left\{f(x) \geqq \alpha G_{a+1} f(x)+1 / n\right\}$ is an $F_{\sigma}$-set. By (3.11), we have for $f=G_{1} h, h \in C_{0}(D)$, and conseqeuntly, for $f=G_{a} h, h \in \boldsymbol{B}\left(D^{*}\right), \alpha>0$, the equality $f(x)=\int_{D^{*} \cup \partial} \mu(x, d y) f(y)$. Therefore,

$$
\begin{aligned}
& \int_{D^{*} \cup \partial} \mu(x, d y) \lim _{\alpha \rightarrow+\infty}\left(\alpha G_{a} f\right)(y)=\lim _{\alpha \rightarrow+\infty} \alpha G_{a} f(x) \\
= & \int_{D^{*} \cup ə} \mu(x, d y) f(y), \quad f \in \boldsymbol{C}_{1} .
\end{aligned}
$$


Using the inequality $\lim _{\alpha \rightarrow+\infty} \alpha G_{a} f \leqq f, f \in \boldsymbol{C}_{1}$ and the criterion above, we can see that $\mu\left(x, \triangle_{0}\right)=0$.

Assertion (iii) is immediate from (3.8) and (3.11).

In the next section, we shall see that $\mu(x, D)=0, x \in \triangle_{0}$.

Let us set $D_{1}^{*}=D^{*}-\triangle_{0}$. By Lemma 3.5 (i), we see $D \subset D_{1}^{*}$. By Lemma 3,4 (i) and Lemma 3. 5 (iii), we have $P_{x}\left(X_{t} \in D^{*}\right.$ for every $\left.t \geqq 0\right)=1, x \in D_{1}$ *. The following two lemmas will assure that the properties stated in Theorem 2 (ii) are valid for $\boldsymbol{X}=\left\{X_{t}, P_{x}, x \in D^{*}\right\}$ except the continuity of the trajectory $X_{t}$ at the boundary $\triangle$.

We call a random time $\sigma \geqq 0$ a Markov time (relative to $\mathfrak{F}_{t}$ ) if, for each $t>0$ and each probability measure $\nu$ on $D^{*}$, the set $\{\sigma<t\}$ is in $\mathfrak{F}_{t}$ up to a set of $P_{\nu}$-measure zero $\left(P_{\nu}(\cdot)=\int_{D^{*}} \nu(d x) P_{x}(\cdot)\right)$. For a Markov time $\sigma$, let $\mathfrak{F}_{\sigma+}$ denote the $\sigma$-field of subsets $\Lambda$ of $\Omega$ such that, for each $t>0$ and each probability measure $\nu$ on $D^{*}, \Lambda \cap\{\sigma<t\}$ is in $\mathfrak{F}_{t}$ up to a set of $P_{\nu}$-measure zero.

\section{Lemma 3.6.}

(i) $\boldsymbol{X}=\left\{X_{t}, P_{x}, x \in D^{*}\right\}$ is a strong Markov process; for each Markov time $\sigma, \Lambda \in \mathfrak{F}_{\sigma+}$ and $f \in \boldsymbol{B}\left(D^{*}\right)$,

$$
E_{x}\left(f\left(X_{\sigma+t}\right) ; \Lambda\right)=E_{x}\left(E_{X_{\sigma}}\left(f\left(X_{t}\right)\right) ; \Lambda\right), \quad x \in D^{*} .
$$

(ii) For each $x \in D^{*}, P_{x}\left(X_{t} \notin \triangle_{0}\right.$ for every $\left.t \geqq 0\right)=1$.

\section{Lemma 3.7.}

(i) Let $\left\{D_{n}\right\}$ be an exhaustion of $D$. Set

$$
\tau_{n}=\inf \left\{t: X_{t} \in D^{*}-D_{n}\right\} \quad \text { and } \quad \tau=\lim _{n \rightarrow+\infty} \tau_{n} .
$$

Then, $P_{x}\left(X_{t}\right.$ is continuous in $\left.0 \leqq t<\tau\right)=1, x \in D^{*}$.

(ii) For each $x \in D$ and Borel set $E$ of $D$,

$$
P_{x}\left(X_{t} \in E, t<\tau\right)=\int_{E} p^{0}(t, x, y) d y .
$$

(iii) For each $x \in D^{*}$,

$$
P_{x}\left(X_{t} \text { is continous for any } t \geqq 0 \text { such that } X_{t} \text { or } X_{t-} \text { is in } D\right)=1 \text {. }
$$

(iv) For each $x \in D^{*}$,

$$
P_{x}\left(X_{t}, X_{t-} \notin \triangle_{0} \text { for every } t \geqq 0\right)=1 .
$$

(v) $\boldsymbol{X}$ is quasi-left continuous; for any sequence of Markov times $\sigma_{n}$ increasing to 


$$
P_{x}\left(\lim _{n \rightarrow+\infty} X_{\sigma_{n}}=X_{\sigma} ; \sigma<+\infty\right)=P_{x}(\sigma<+\infty), \quad x \in D^{*}
$$

Proof of Lemma 3. 6 (i). Since $X_{t}$ is a modification of $\tilde{X}_{t}$, relations (3. 9) and (3.10) hold for $\boldsymbol{X}$, if we replace $\tilde{X}_{t}$ there with $X_{t}$.

Take a Markov time $\sigma$ and a set $\Lambda \in \mathfrak{F}_{\sigma+}$. The Markov property (3. 10) for $X_{t}$ and a usual limiting procedure lead us to

$$
\begin{aligned}
& E_{x}\left(G_{1} f\left(X_{\sigma}\right) ; \Lambda\right) \\
& =E_{x}\left(\int_{\sigma}^{+\infty} e^{-\infty(t-\sigma)}\left(f\left(X_{t}\right)+(\alpha-1) G_{1} f\left(X_{t}\right) d t ; \Lambda\right),\right.
\end{aligned}
$$

for $f \in C_{0}(D), x \in D^{*}$. Here, we have used the resolvent equation, the right continuity of $X_{t}$ in $t \geqq 0$ and the continuity of $G_{1} f(x), f \in C_{0}(D)$ in $x \in D^{*}$. Since $P_{x}\left(X_{t} \in \triangle\right)=0, x \in D^{*}, t>0$, we can see that equation (3. 12) holds also for $f \in \boldsymbol{B}\left(D^{*}\right)$. By setting $f=G_{\infty} h . h \in \boldsymbol{B}(D), \alpha>0$, in equation (3. 12), we have $E_{x}\left(G_{\infty} G_{1} h\left(X_{\sigma}\right) ; \Lambda\right)=E_{x}\left(\int_{\sigma}^{+\infty} e^{-\infty(t-\sigma)} G_{1} h\left(X_{t}\right) d t ; \Lambda\right)$. By the resolvent equation (G. 3) and (G. 3)' (Lemma 3. 2), we have, for $\beta>0$ and $f \in \boldsymbol{C}\left(D^{*}\right)$,

$$
\begin{aligned}
& E_{x}\left(G_{\omega}\left(\beta G_{\beta} f\right)\left(X_{\sigma}\right) ; \Lambda\right)=E_{x}\left(\int_{\sigma}^{+\infty} e^{-\infty(t-\sigma)}\left(\beta G_{\beta} f\right)\left(X_{t}\right) d t ; \Lambda\right) \\
= & E_{x}\left(\int_{\sigma}^{+\infty} e^{-\omega(t-\sigma)}\left(\beta G_{\beta} f\right)\left(X_{t}\right) \chi_{D}\left(X_{t}\right) d t ; \Lambda\right) .
\end{aligned}
$$

Letting $\beta$ tend to infinity, we have, by Theorem 1 (iii),

$$
\begin{aligned}
& E_{x}\left(G_{\alpha} f\left(X_{\sigma}\right) ; \Lambda\right) \\
= & E_{x}\left(\int_{0}^{+\infty} e^{-\alpha t} f\left(X_{\sigma+t}\right) d t ; \Lambda\right), \quad \alpha>0, \quad f \in C\left(D^{*}\right), \quad x \in D^{*},
\end{aligned}
$$

which proves conclusion (i) of Lemma 3. 6.

Proof of Lemma 3. 6 (ii).

Here, we can go along the same line as in H. Kunita and T. Watanabe [11], Section 2, (j). Set, for $A \subset D^{*}$,

$$
\begin{aligned}
\sigma_{A} & =\inf \left\{t>0 ; X_{t} \in A\right\}, \\
& =+\infty, \text { if there is no such } t .
\end{aligned}
$$

$\sigma_{A}$ is a Markov time if $A$ is open or closed. Since $\triangle_{0}$ is an $F_{\sigma}$-set (Lemma 3. 5 (ii)), Lemma 3.5 (ii) and the strong Markov property will imply the second assertion of Lemma 3. 6 .

Proof of Lemma 3. 7 (i), (ii).

It follows from Lemma 2. 11 (iv), that, for each compact set $K \subset D$ and $\varepsilon>0$, 
(3. 14) $\lim _{t \uparrow 0} \frac{1}{t} \sup _{x \in \mathbb{K}} p\left(t, x, D-U_{\varepsilon}(x)\right)=0$.

where $U_{\varepsilon}(x)=\{y \in D, \rho(x, y)<\varepsilon\}$.

(3. 14) implies

(3. 15) $\quad P_{x}\left(X_{t}\right.$ is continuous for every $\left.t<\tau_{n}\right)=1$,

$x \in D^{*}$, (see E.B. Dynkin [3], Lemma 6.6). Letting $n$ tend to infinity, we have the first statement of Lemma 3.7.

Next, take a regular exhaustion $\left\{D_{n}\right\}$. Then, we have

$$
P_{x}\left(\tau_{n}=0\right)=1, \quad x \in \partial D_{n}, \quad n=1,2, \cdots,
$$

(3.17) for each $n$ and compact set $K \subset D_{n}$,

$$
\lim _{u \downarrow 0} \sup _{x \in \mathcal{K}} P_{n}\left(\tau_{n} \leqq u\right)=0
$$

(3.18) for each twice continuously differentiable functions $f$ on $D$,

$$
\lim _{t \downarrow 0} \frac{1}{t}\left(T_{t} f(x)-f(x)\right)=\frac{1}{2} \triangle f(x), \quad x \in D .
$$

Indeed, (3. 18) is immediate. Property (3.16) follows from $P_{x}\left(\tau_{n}>t\right) \leqq 1-P_{x}$ $\left(X_{t} \in D-D_{n}\right)$ and $P_{x}\left(X_{t} \in D^{*}-D_{n}\right) \geqq \int_{D-D_{n}} p^{0}(t, x, y) d y$. Property (3.17) follows from the following estimate ([3], Lemma 6.1): for any Borel subset $G$ of $D$, $P_{x}\left(X_{t} \in D_{n} \cup \partial D_{n}\right.$ for every $\left.t \leqq u\right) \geqq p(u, x, G)-\sup _{y \in D-D_{n}, 0<t \leqq u} p(t, y, G)$. Since $T_{t}$ maps $\boldsymbol{B}(D)$ into $\boldsymbol{C}(D)$ (Theorem $1(\mathrm{v})$ ), it follows from (3.16) and (3.17) that the operator $T_{t}^{n}$, defined by $T_{t}^{n} f(x)=E_{x}\left(f\left(X_{t}\right) ; t<\tau_{n}\right), x \in D_{n}$, makes invariant the space of all continuous functions which vanish on $\partial D_{n}$ (see E.B. Dynkin [4]. Theorem 13.1 and Theorem 13.8). Let $p^{(n)}(t, x, y)$ denote the transition density of the absorbing barrier Brownian motion on $D_{n}$. Then, combining the above property of $T_{t}^{n}$, the continuity of trajectory $X_{t}, t<\tau$, and formula (3. 18), we can conclude ([4], chap. V, §6) that, for any Borel subset $E$ of $D_{n}$,

$$
P_{x}\left(X_{t} \in E, t<\tau_{n}\right)=\int_{E} p^{(n)}(t, x, y) d y, t>0, \quad x \in D_{n} .
$$

Let $n$ tend to infinity to obtain conclusion (ii) of our lemma.

Proof of Lemma 3.7 (iii), (iv).

Let us fix $c>0$. Denote by $\mathbb{Q}$ the class of all $D^{*}$-valued functions defined on $[0, c]$. Define the operator $q$ from $\mathbb{Q}$ to $\mathbb{Q}$ by $q \varphi(t)=\varphi(c-t), 0 \leqq t \leqq c, \varphi \in \mathbb{Q}$, For $\omega \in \Omega$, we define $\nu(\omega)=\left\{X_{t}(\omega) ; 0 \leqq t \leqq c\right\}$. 
$\nu(\omega) \in \mathbb{Q}$ for almost all $\omega\left(P_{x}\right)$. We set for $A \in \mathfrak{F}_{c} \gamma A=\nu^{-1} \mathfrak{q} \nu A$. According to the symmetry and the conservativity of $p(t, x, y)$, it is easy to see that

$$
\int_{D} P_{x}(\gamma A) d x=\int_{D} P_{x}(A) d x, \quad A \in \mathfrak{F}_{c}
$$

We shall first prove assertion (iv).

Put $A_{h}^{c+h}=\left\{\omega ; X_{t-} \in \triangle_{0}\right.$ for some $\left.t \in(h, c+h)\right\}$ and $B_{0}^{c}=\left\{\omega ; X_{t} \in \triangle_{0}\right.$ for some $\left.t \in(0, c)\right\}, h \geqq 0$.

Obviously, $A_{0}^{c}=\gamma B_{0}^{c}$, and by Lemma 3.6 (ii), and (3. 19), we have $\int_{D} P_{x}\left(A_{0}^{c}\right) d x$ $=\int_{D} P_{x}\left(B_{0}^{c}\right) d x=0$. Hence, $P_{x}\left(A_{0}^{c}\right)=0$ for almost all $x \in D$. By (3. 10), we see, for each $x \in D^{*}, P_{x}\left(A_{h}^{c+h}\right)=\int_{D} p(h, x, y) P_{y}\left(A_{0}^{c}\right) d y=0$. Letting $c$ tend to infinity and then $h$ tend to zero, we obtain conclusion (iv) of the present lemma.

Coming to the proof of assertion (iii), consider the set $\widehat{A}_{0}^{c}=\left\{\omega ; X_{t-} \in D\right.$, $X_{t-} \neq X_{t}$ for some $\left.t \in(0, c)\right\}$. Then, $\widetilde{A}_{0}^{c}=A_{1} \cup A_{2}$, where, $A_{1}=\left\{\omega ; X_{t-} \in D\right.$, $X_{t} \in D, X_{t} \neq X_{t-}$ for some $\left.t \in(0, c)\right\}$ and $A_{2}=\left\{\omega ; X_{t-} \in D, X_{t} \in \triangle\right.$ for some $t \in(0, c)\}$. Denote by $S$ a countable dense subset of $(0, c)$. Obviously, $A_{1} \subset \bigcup_{s \in S}\left\{\omega ; X_{s} \in D, X_{t}\right.$ has a discontinuity for some $\left.t \in\left(s,\left(s+\tau\left(\theta_{s} \omega\right)\right) \wedge c\right)\right\}$ and $A_{2} \subset \bigcup_{s \in S}\left\{\omega ; X_{s} \in D, X_{\tau_{n}\left(\theta_{s} \omega\right)} \notin \partial D_{n}\right.$ for some $n$ such as $\left.s+\tau_{n}\left(\theta_{s} \omega\right)<c\right\}$. By virtue of (i) and (ii) of Lemma 3.7, one has $P_{x}\left(A_{1} \cup A_{2}\right)=0$ for $x \in D$, and consequently (see the proof of (iv)) for all $x \in D^{*}$. Set $\widetilde{B}_{0}^{c}=\gamma \widetilde{A}_{c}^{0}$, then the same argument as in the proof of (iv) leads to $P_{x}\left(\widetilde{B}_{0}^{c}\right)=0, x \in D^{*}$.

The final statement of Lemma 3. 7 follows from assertion (iv) of the lemma and assertion (i) of Lemma 3. 6. (see [11], Section 2, (i)).

\section{The Dirichlet norm related to the process and the continuity of trajectories at the boundary}

The main purpose of this section is to show in Lemma 4. 5 that, for almost all $\omega$, the entire trajectory $X_{t}(\omega), 0 \leqq t<+\infty$, is continuous. Since we already proved that $X_{t}(\omega)$ is continuous for all $t>0$ such that $X_{t}(\omega)$ or $X_{t-}(\omega) \in D$, it remains to prove that $X_{t}(\omega)$ has no jumps at the boundary $\triangle$.

First, we will give an integral representation of 1 -excessive functions.

Definition 6. A non-negative function $u$ on $D^{*}$ is called $\alpha$-excessive if

$$
e^{-a t} T_{t} u(x) \uparrow u(x) \text { as } t \downarrow 0 \text { for each } x \in D^{*} .
$$

\section{Lemma 4.1.}

(i) If a non-negative function $u$ defined on $D$ satisfies (4. 1) for every $x \in D$, then $u$ is uniquely extended to an $\alpha$-excessive function on $D^{*}$. 
(ii) If $u_{1}$ and $u_{2}$ are $\alpha$-excessive and $u_{1}(x)=u_{2}(x)$ almost everywhere on $D$, then $u_{1}$ and $u_{2}$ coincide on $D^{*}$.

Proof. (i). For $x \in D^{*}, e^{-a t} T_{t} u(x)=e^{-\infty t} \int_{D} p(t, x, y) u(y) d y$ is monotone increasing as $t \downarrow 0$, and we have only to set $\tilde{u}(x)=\lim _{t \downarrow 0} T_{t} u(x)$. The uniqueness of $\tilde{u}$ and assertion (ii) are easily verified.

Set $\triangle_{1}=\triangle-\triangle_{0}$.

\section{Lemma 4.2.}

(i) $G_{a}(x, y),(x, y) \in D^{*} \times D$, can be extended to $(x, y) \in D^{*} \times D^{*}$ in such a way that the extended function $G_{o s}(x, y)$ is symmetric in $x, y \in D^{*}$ and, for each $x($ resp. $y) \in D^{*}$, it is $\alpha$-excessive in $y($ resp. $x)$.

(ii) For each branching point $x \in \triangle_{0}$, the branching measure $\mu(x, \cdot)$ is concentrated on $\triangle_{1} \cup \partial$.

Proof. (i). By Theorem 1 (v) and Lemma 3.3, $G_{a}(x, y)$ is, for each $y \in D$, $\alpha$-excessive in $x \in D^{*}$ and it satisfies (4.1) as a function of $y \in D$, for each $x \in D^{*}$. By virtue of Lemma 4.1, $G_{a}(x, y), x \in D^{*}$, has an $\alpha$-excessive extension with respect to $y$. The symmetry of the extended kernel follows from Theorem 1 (ii). (ii). As we have seen in Section 3, (see the proof of Lemma $3.5)$,

$$
f(x)=\int_{D \cup \triangle_{1}} \mu(x, d y) f(y), \text { for } f=G_{o s} h, h \in \boldsymbol{B}\left(D^{*}\right) .
$$

Hence, by Lemma 4.1 (ii),

$$
G_{a}(x, y)=\int_{D \cup \triangle_{1}} \mu(x, d z) G_{a}(z, y), \quad y \in D .
$$

When $x \in \triangle_{0}, G_{o}(x, y)$ is $\alpha$-harmonic in $y$ and equation (4.2) implies that $\mu(x, \cdot)$ has no mass on $D$ (see Lemma 2.1).

\section{Theorem 3.}

If $u$ is 1-excessive and $\int_{D} u(x) d x<+\infty$, then there exists a unique measure $\nu$ concentrated on $D \cup \triangle_{1}$ such as

$$
u(x)=\int_{D \cup \triangle_{1}} G_{1}(x, y) \nu(d y), \quad x \in D^{*} .
$$

We call $\nu$ the canonical measure corresponding to $u$.

Proof. Since $u$ is 1 -excessive, there is an increasing sequence of nonnegative functions $f_{n}, n=1,2, \cdots$, such that 


$$
G_{1} f_{n}(x) \underset{n \rightarrow+\infty}{\uparrow} u(x), \quad x \in D^{*} .
$$

Because of Theorem 1 (ii), $\int_{D} f_{n}(x) d x=\left(f_{n}, G_{1} 1\right)=\left(G_{1} f_{n}, 1\right) \leqq \int_{D} u(x) d x<+\infty$. Hence, extracting a subsequence if necessary, the sequence of measures $f_{n}(x) d x$ converges weakly to a measure $\nu_{0}(d x)$ on $D^{*}$. By Corollary to Lemma 3. 1, $G_{1} \varphi$ is continuous if $\varphi \in C_{0}(D)$, so that $(\varphi, u)=\lim _{n \rightarrow+\infty}\left(\varphi, G_{1} f_{n}\right)=\lim _{n \rightarrow+\infty}\left(G_{1} \varphi, f_{n}\right)$ $=\int_{D \cup \triangle} G_{1} \varphi(x) \nu_{0}(d x), \varphi \in C_{0}(D)$. Thus, it holds that

$$
u(x)=\int_{D \cup \triangle} G_{1}(x, y) \nu_{0}(d y),
$$

for almost all $x \in D$, and consequently (Lemma 4 . 1 (ii)) for every $x \in D^{*}$. Using (4. 2) and Lemma 4. 2 (ii), we can rewrite (4.4) in the form (4.3) with $\nu$ defined by $\nu(d y)=\nu_{0}(d y)+\int_{\triangle_{0}} \nu_{0}(d z) \mu(z, d y)$. The measure $\nu$ of (4.3) is uniquely determined by $u$. In fact, for any $f \in \boldsymbol{C}\left(D^{*}\right), \int_{D^{*}} f(x) \nu(d x)=\lim _{\alpha \rightarrow+\infty} \alpha \int_{D \cup \triangle_{1}} G_{a f} f(x) \nu(d x)$ $=\lim _{\alpha \rightarrow+\infty} \alpha \int_{D \cup \triangle_{1}}\left(G_{1} f(x)-(\alpha-1) G_{1} G_{\alpha} f(x)\right) \nu(d x)=\lim _{\alpha \rightarrow+\infty} \alpha\left(u, f-(\alpha-1) G_{o} f\right) . \quad$ The proof of Theorem 3 is complete.

Our next task is about the canonical measures corresponding to a special class of excessive functions.

Definition 7. The $(-\infty,+\infty]$-valued function $A_{t}(\omega)$ on $[0,+\infty] \times \Omega$ is called an $\alpha$-additive functional of $\boldsymbol{X}$, if

(A. 1) for fixed $t, A_{t}(\omega)$ is $\mathfrak{\mho}_{t+}$-measurable in $\omega$,

and if there is $\mathfrak{A}$-measurable set $\Omega_{A}$ closed under the operation $\theta_{t}, t>0$, such that $P_{x}\left(\Omega_{A}\right)=1, x \in D^{*}$, and for each fixed $\omega \in \Omega_{A}$,

(A. 2) $A_{t}(\omega)$ is right continuous and has the left limit in $t$,

(A. 3) $\zeta(\omega)=0$ implies $A_{t}(\omega)=0$ for $t \geqq 0$,

where $\zeta(\omega)$ is a hitting time to $\partial$, and

(A. 4) $A_{t+s}(\omega)=A_{t}(\omega)+e^{-a t} A_{s}\left(\theta_{t} \omega\right)$, for $t, s \geqq 0$.

Two $\alpha$-additive functionals $A$ and $B$ are called equivalent and denoted by $A \approx B$, when $A_{t}=B_{t}$ holds almost everywhere $\left(P_{x}\right)$ for each $t \geqq 0$ and $x \in D^{*}$. A 0 -additive functional will be called an additive functional simply.

Put $\mathfrak{R}=\left\{u ; u=G_{o} f, f \in \boldsymbol{B}\left(D^{*}\right)\right\} . \quad \Re$ is contained in $\boldsymbol{B}\left(D^{*}\right)$ and independent of $\alpha>0$. If $G_{o s} f_{1}(x)=G_{o s} f_{2}(x), x \in D^{*}, f_{1}, f_{2} \in \boldsymbol{B}\left(D^{*}\right)$, then, as one easily sees, 
$f_{1}=f_{2}$ almost every-where on $D$.

Take $u \in \Re$. If $u=G_{1 / 2} f, f \in \boldsymbol{B}\left(D^{*}\right)$, we set

$$
A_{t}^{u}=e^{-t / 2} u\left(X_{t}\right)-u\left(X_{0}\right)+\int_{0}^{t} e^{-s / 2} f\left(X_{s}\right) d s, \quad t \geqq 0 .
$$

It is easy to see that $A_{t}^{u}$ is a $1 / 2$-additive functional and it is uniquely determined by $u$ up to equivalence. Clearly $E_{x}\left(A_{t}^{u}\right)=0, x \in D^{*}, t \geqq 0$. We see that

$$
v_{u}(x)=E_{x}\left(\left(A_{+\infty}^{u}\right)^{2}\right)
$$

is a 1-excessive function. In fact, $A_{+\infty}^{u}(\omega)=A_{t}^{u}(\omega)+e^{-t / 2} A_{+\infty}^{u}\left(\theta_{t} \omega\right)$ implies $v_{u}(x)$ $=E_{x}\left(\left(A_{t}^{u}\right)^{2}\right)+2 E_{x}\left(e^{-t / 2} A_{t}^{u} E_{X_{t}}\left(A_{+\infty}^{u}\right)\right)+E_{x}\left(e^{-t} E_{X_{t}}\left(\left(A_{+\infty}^{u}\right)^{2}\right)=E_{x}\left(\left(A_{t}^{u}\right)^{2}\right)+e^{-t} T_{t} v_{u}(x)\right.$, and $e^{-t} T_{t} v_{u}(x) \uparrow v_{u}(x)$ as $t \downarrow 0, x \in D^{*}$. Moreover, $\int_{D} v_{u}(x) d x<+\infty$, and so, $v_{u}$ is expressed as the $G_{1}$-potential of a measure on $D_{1}^{*}=D \cup \triangle_{1}$ according to Theorem 3.

Definition 8. For $u \in \Re$, define $A_{t}^{u}$ and $v_{u}$ by (4.5) and (4.6), respectively. Denote by $\nu_{u}$ the canonical measure on $D \cup \triangle_{1}$ corresponding to $v_{u}$. Set $\|u\|_{\boldsymbol{X}}=\sqrt{\nu_{u}\left(D \cup \triangle_{1}\right)}$ and call this the Dirichlet norm of $u \in \Re$ with respect to the process $\boldsymbol{X}$.

We will show

Theorem 4. Let $u$ be in $\Re$. Then,

(i ) $\|u\| \|_{X}^{2}=\int_{D}(\operatorname{grad} u, \operatorname{grad} u)(x) d x$,

(ii) $\nu_{u}\left(\triangle_{1}\right)=0$.

Let us prepare two lemmas.

\section{Lemma 4.3.}

\|\|$u \|_{X}^{2}=2(u, f)-(u, u), \quad u \in \Re$.

Proof. Since $\int_{D} G_{1}(x, y) d x=\int_{D} G_{1}(y, x) d x=q(y)=1$ for $y \in D \cup \triangle_{1}$ (Lemma 3.5 (iii)), we have $\|u\|_{X}^{2}=\nu_{u}\left(D \cup \triangle_{1}\right)=\int_{D} v_{u}(x) d x$. On the other hand,

$$
\begin{aligned}
v_{u}(x) & =E_{x}\left(\left(\int_{0}^{+\infty} e^{-s / 2} f\left(X_{s}\right) d s\right)^{2}\right)-u(x)^{2} \\
& =2 E_{x}\left(\int_{0}^{+\infty} e^{-t / 2} f\left(X_{t}\right) d t \int_{t}^{+\infty} e^{-s / 2} f\left(X_{s}\right) d s\right)-u(x)^{2} \\
& =2 E_{x}\left(\int_{0}^{+\infty} e^{-t} f\left(X_{t}\right) d t E_{X_{t}}\left(\int_{0}^{+\infty} e^{-s / 2} f\left(X_{s}\right) d s\right)\right)-u(x)^{2} \\
& =2 \int_{D} G_{1}(x, y) f(y) u(y) d y-u(x)^{2}
\end{aligned}
$$


Hence, Lemma 4.3 is valid.

Lemma 4.4. Let $\tau$ be the first exit time from $D$ defined in Lemma 3.7 (i). Then we have, for $u \in \Re$,

$$
\begin{aligned}
& E_{x}\left(\left(A_{\tau-}^{u}\right)^{2}\right)=\int_{D} G_{1}^{0}(x, y)(\operatorname{grad} u, \operatorname{grad} u)(y) d y, \quad x \in D, \\
& E_{x}\left(\left(A_{\tau_{-}}^{u}\right)^{2}\right)=\int_{D} G_{1}^{0}(x, y) \nu_{u}(d y), \quad x \in D, \\
& \nu_{u}(D)=\int_{D}(\operatorname{grad} u, \operatorname{grad} u)(y) d y .
\end{aligned}
$$

Proof. Let $\left\{\tau_{n}\right\}$ be the first exit times from an exhaustion $\left\{D_{n}\right\}$ of $D$. By definition, $\tau_{n} \uparrow \tau$. In view of Lemma 3.7 (ii), $\left\{X_{t}, t<\tau_{n}\right\}$ is equivalent to the absorbing barrier standard Brownian motion on $D_{n}$. Now, suppose that $f$ belongs to $\boldsymbol{C}^{1}(D)$. Then, $u=G_{1 / 2} f=G_{1 / 2}^{0} f+R_{1 / 2} f$ belongs to $\boldsymbol{C}^{2}(D)$ and $\left(\frac{1}{2}-\frac{1}{2} \triangle\right) u(x)=f(x), x \in D^{15)}$. Applying the formula concerning stochastic integrals $^{16)}$ to the function $F(t, x)=e^{-t / 2} u(x)$, we obtain $A_{\tau_{n}}^{u}=\int_{0}^{\tau_{n}} e^{-s / 2} \operatorname{grad} u\left(X_{s}\right) d X_{s}$, and consequently

$$
E_{x}\left(\left(A_{\tau_{n}}^{u}\right)^{2}\right)=E_{x}\left(\int_{0}^{\tau_{n}} e^{-s}(\operatorname{grad} u, \operatorname{grad} u)\left(X_{s}\right) d s\right), \quad x \in D
$$

Consider the collection $\mathfrak{S}$ of all bounded functions $f$ on $D$ such that $u=G_{1 / 2} f$ satisfies equation (4.10) for a fixed $n$. Obviously $\mathfrak{S}$ is a linear space and $C^{1}(D)$ $\subset \mathfrak{S}$. It is easy to see that, if $f_{\boldsymbol{k}} \in \mathfrak{S}$ converges boundedly to a bounded function $f$, then $f \in \mathfrak{S}$. Hence, $\mathfrak{S}=\boldsymbol{B}(D)$. We get formula (4.7) by letting $n$ tend to infinity in (4.10). In order to show identity (4.8), we have only to let $n$ tend to infinity in the first and last term of the following identity.

$$
\begin{aligned}
& E_{x}\left(\left(A_{\tau_{n}}^{u}\right)^{2}\right)=v_{u}(x)-E_{x}\left(e^{-\tau} n v_{u}\left(X_{\tau_{n}}\right)\right) \\
& =\int_{D \cap \triangle_{1}} G_{1}(x, y) \nu_{u}(d y)-\int_{D \cup \triangle_{1}} E_{x}\left(e^{-\tau} G_{1}\left(X_{\tau_{n}}, y\right)\right) \nu_{u}(d y) \\
& =\int_{D}\left(G_{1}(x, y)-E_{x}\left(e^{-\tau} G_{1}\left(X_{\tau_{n}}, y\right)\right)\right) \nu_{u}(d y) \text {. }
\end{aligned}
$$

The formulac (4.7) and (4.8) imply identity (4.9).

Proof of Theorem 4. It follows from the definition of $R_{1 / 2}(x, y)$ that, when $u \in \Re$ and $u=G_{1 / 2} f, f \in \boldsymbol{B}(D)$,

15) $\boldsymbol{C}^{1}(D)\left(C^{2}(D)\right)$ is the aggregate of all bounded, continuously (resp. twice continuously) differentiable functions on $D$.

16) cf. [4], (7. 77). 


$$
\boldsymbol{D}_{1 / 2}(u, u)=2(u, f) .
$$

Indeed, the same procedure as in the proof of Lemma 2.10 is applicable to get $\boldsymbol{D}_{1 / 2}\left(R_{1 / 2} f, R_{1 / 2} f\right)=2\left(R_{1 / 2} f, f\right)$. It is easy to see that $\boldsymbol{D}_{1 / 2}\left(G_{1 / 2}^{0} f, G_{1 / 2}^{0} f\right)$ $=2\left(G_{1 / 2}^{0} f, f\right)$ and $\boldsymbol{D}_{1 / 2}\left(G_{1 / 2}^{0} f, R_{1 / 2} f\right)=0$. Rewrite (4.11) in the form, $2(u, f)-(u, u)$ $=\int_{D}(\operatorname{grad} u, \operatorname{grad} u)(y) d y$. Now, assertions (i) and (ii) of Theorem 4 follow from Lemma 4.3 and Lemma 4.4, respectively.

Coming to our main task about the continuity of trajectories of $\boldsymbol{X}$, we shall introduce several notations and concepts given by M. Motoo and S. Watanabe [18]. In [18], Hunt processes are treated. Our process $\boldsymbol{X}$ is not a Hunt process in general: It may include branching points. However, owing to Lemmas 3.6, 3.7 and 4.1, all the results in [18] can be applied to our process.

Set

$\mathfrak{C}_{1}^{+}=\left\{A ; A\right.$ is an additive functional of $\boldsymbol{X}$ such that $A_{t}(\omega), t \geqq 0, \omega \in \Omega_{A}$, is nonnegative, continuous in $t$ and $E_{x}\left(A_{t}\right)<+\infty$ for $\left.t \geqq 0, x \in D^{*}\right\}^{17)}$

$\mathfrak{\Im}_{1}=\left\{A ; A=A_{1}-A_{2}, A_{i} \in \mathfrak{F}_{1}^{+}, i=1,2\right\}$,

$\mathfrak{M}=\left\{A ; A\right.$ is an additive functional of $\boldsymbol{X}$ such that $E_{x}\left(A_{t}^{2}\right)<+\infty$ and $E_{x}\left(A_{t}\right)$ $=0$ for $\left.t \geqq 0, x \in D^{*}\right\}$.

Let $A, B \in \mathfrak{M}$. Then there exists a unique element of $\mathfrak{E}_{1}$, denoted by $\langle A, B\rangle$, satisfying the following condition: $E_{x}\left(\langle A, B\rangle_{t}\right)=E_{x}\left(A_{t} B_{t}\right)$ holds for every $t \geqq 0$ and $x \in D^{*}$. For $A \in \mathfrak{M},\langle A, A\rangle$ will be denoted by $\langle A\rangle$. It is an element of. $\mathfrak{S}_{1}^{+}$.

We set, for $A \in \mathfrak{M}$,

$\boldsymbol{L}^{2}(A)=\left\{f ; f\right.$ is a measurable function on $D^{*}$ such that $E_{x}\left(\int_{0}^{t} f\left(X_{s}\right)^{2} d\langle A\rangle_{s}\right)<+\infty$ for every $\left.t>0, x \in D^{*}\right\}$.

\section{Definition 9.}

Let $A \in \mathfrak{M}$ and $f \in \boldsymbol{L}^{2}(A) . \quad B \in \mathfrak{M}$ is called the stochastic integral of $f$ by $A$ and is denoted by $B=\int f d A$ if $E_{x}\left(B_{t} C_{t}\right)=E_{x}\left(\int_{0}^{t} f\left(X_{s}\right) d\langle A, C\rangle_{s}\right), t \geqq 0$, holds for every $C \in \mathfrak{M}$.

The stochastic integral exists uniquely for $A \in \mathfrak{M}$ and $f \in \boldsymbol{L}^{2}(A)$ (Theorem 10.4 of [18]). As a consequence of Theorem 4, we have

Theorem 5. Denote by $\chi_{\triangle_{1}}$ the indicator function of the set $\triangle_{1}$. It holds that $\int \chi_{\triangle_{1}} d A \approx 0$ for any $A \in \mathfrak{M}$.

17) $\Omega_{A}$ is a suitable defining set of $A$ (see Definition 7). 
Proof. (i). Set, for $u \in \Re$ and $u=G_{1 / 2} f$ with $f \in \boldsymbol{B}\left(D^{*}\right)$,

$$
\widehat{A}_{t}^{u}=u\left(X_{t}\right)-u\left(X_{0}\right)+\int_{0}^{t}\left(f\left(X_{s}\right)-\frac{1}{2} u\left(X_{s}\right)\right) d s, t \geqq 0 .
$$

Obviously, $\widehat{A}^{u} \in \mathfrak{M}$. Let us show, for $u \in \mathfrak{R}$,

$$
\int \chi_{\triangle_{1}} d \widehat{A}^{u} \approx 0, \text { or equivalently }
$$

$$
\int_{0}^{t} \chi_{\triangle_{1}}\left(X_{s}\right) d\left\langle\widehat{A}^{u}\right\rangle_{s}=0, t \geqq 0, \text { almost everywhere }\left(P_{x}\right), x \in D^{*} \text {. }
$$

Since $A^{u}$ defined by (4.5) is related to $\widehat{A}^{u}$ by $A_{t}^{u}=e^{-t / 2} \widehat{A}_{t}^{u}+\frac{1}{2} \int_{0}^{t} e^{-s / 2} \widehat{A}_{s}^{u} d s, v_{u}$ defined by (4.6) is expressed as

$$
v_{u}(x)=E_{x}\left(\int_{0}^{+\infty} e^{-s} d\left\langle\widehat{A}^{u}\right\rangle_{s}\right), \quad x \in D^{*}
$$

On the other hand, $v_{v}(x)=\int_{D \cup \triangle_{1}} G_{1}(x, y) \nu_{u}(d y)$, and by virtue of Theorem 4 (which states $\nu_{u}\left(\triangle_{1}\right)=0$ ), $\left\langle\widehat{A}^{u}\right\rangle_{t}$ can never increase when $X_{t} \in \triangle_{1}$ (see [6] or [14]), that is, $\int \chi_{\triangle_{1}}\left(X_{s}\right) d\left\langle\widehat{A}^{u}\right\rangle_{s} \approx 0$.

(ii). In order to derive Theorem 5 from (4.13), we introduce several notations. We write 1.i.m $A^{n}=A$, for $A^{n}$ and $A \in \mathfrak{M}$, if and only if $E_{x}\left(\left(A_{t}^{u}-A_{t}\right)^{2}\right)$ $\underset{n \rightarrow+\infty}{\longrightarrow} 0, x \in D^{*}, t \geqq 0$. A subset $\boldsymbol{L}$ of $\mathfrak{M}$ is called a subspace, if $\boldsymbol{L}$ satisfies the following conditions.

(a) If $A, B \in \boldsymbol{L}$, then $A+B \in \boldsymbol{L}$.

(b) If $A^{n} \in \boldsymbol{L}$ and $A=1$. i.m $A^{n}$, then $A \in \boldsymbol{L}$.

(c) If $A \in \boldsymbol{L}$ and $f \in \boldsymbol{L}^{2}(A)$, then $\int f d A \in \boldsymbol{L}$.

For a subset $\boldsymbol{M}$ of $\mathfrak{M}, \boldsymbol{L}(\boldsymbol{M})$ will stand for the minimum subspace which contains $\boldsymbol{M}$. We note that, Theorem 12.2 of [18] states $\mathfrak{M}=\boldsymbol{L}\left(\widehat{A}^{u} ; u \in \boldsymbol{R}\right)$, where $\widehat{A}^{u}$ is defined by (4.12). If we set $\mathfrak{M}^{\prime}=\left\{A ; A \in \mathfrak{M}, \int \chi_{\triangle_{1}} d A \approx 0\right\}$, then $\mathfrak{M}^{\prime}$ is a subspace of $\mathfrak{M}$ and contains $\widehat{A}^{u}, u \in \mathfrak{R}$, by (4.13). Hence $\mathfrak{M}^{\prime}=\mathfrak{M}$, completing the proof of Theorem 5 .

By the following lemma, we will complete the proof of Theorem 2 stated in Section 1 .

Lemma 4.5. The strong Markov process $X=\left\{X_{t}, \mathfrak{F}_{t+}, P_{x}, x \in D^{*}\right\}$ is a diffusion, that is, $\boldsymbol{X}$ satisfies the condition

(b) $P_{x}\left(X_{t}\right.$ is continuous for every $\left.t \geqq 0\right)=1, x \in D^{*}$. 
Proof. Let $\rho(x, y)$ be the metric on $D^{*}$ defined by (3.1). We shall set, for convenience, $\rho(x, \partial)=+\infty, x \in D^{*}$ and $\rho(\partial, \partial)=0$. For $\varepsilon>0$, define $\sigma^{\varepsilon}$ by

$$
\begin{array}{rlrl}
\sigma^{\varepsilon} & =\inf \left\{t ; \rho\left(X_{t-},\right.\right. & \left.\left.X_{t}\right)>\varepsilon\right\}, \\
& =+\infty & & \text { if there is no such } t,
\end{array}
$$

and $\sigma_{1}^{\varepsilon}, \sigma_{2}^{\varepsilon}, \cdots$, by $\sigma_{1}^{\varepsilon}=\sigma^{\varepsilon}, \sigma_{n}^{\varepsilon}=\sigma_{n-1}^{\varepsilon}(\omega)+\sigma^{\varepsilon}\left(\theta_{\sigma_{n-1}^{\varepsilon}} \omega\right)$. Set $\mathfrak{p}_{t}^{\varepsilon, E}=\sum_{\sigma_{n}^{\varepsilon} \leqq t} \chi_{E}\left(X_{\sigma_{n}}^{\varepsilon}\right)$, for $E \in \mathfrak{B}\left(D^{*} \cup \partial\right)$ and $t \geqq 0$. Obviously, $\mathfrak{p}^{\varepsilon} i^{E}$ is an additive functional. We shall denote $\mathfrak{p}_{t}^{\varepsilon, D^{*} \cup \partial}$ by $\mathfrak{p}_{t}^{\varepsilon}$. Statement (b) is equivalent to

$$
\mathfrak{p}_{t}^{\varepsilon} \approx 0, \text { for any } t \geqq 0 \text { and } \varepsilon>0 \text {. }
$$

Let us show (4.16). We can find $B_{m} \in \mathfrak{B}\left(D^{*} \cup \partial\right)$ such that $B_{m} \uparrow D^{*} \cup \partial$ and $E_{x}\left(\mathfrak{p}_{t}^{\mathbb{\varepsilon}, B_{m}}\right)<+\infty, x \in D^{*}, t \geqq 0\left(\right.$ Lemma 3.1 of [22]). For $B_{m}$, there is $\tilde{\mathfrak{p}}_{t}^{\mathfrak{\varepsilon}, m} \in \mathfrak{E}_{1}^{+}$ such as

$$
E_{x}\left(\mathfrak{p}_{t}^{\varepsilon, B_{m}}\right)=E_{x}\left(\tilde{\mathfrak{p}}_{t}^{\mathfrak{\varepsilon}, m}\right), \quad t \geqq 0, \quad x \in D^{*} .
$$

If we put $\mathfrak{q}_{t}^{\varepsilon, m}=\mathfrak{p}_{t}^{\varepsilon, B_{m}}-\mathfrak{\mathfrak { p }}_{t}^{\varepsilon, m}$, then $\mathfrak{q}^{\varepsilon, m} \in \mathfrak{M}$ and

(4. 18) $\left\langle\mathfrak{q}^{\varepsilon, m}\right\rangle \approx \mathfrak{p}^{\varepsilon, m}$ (Theorem 2.2 of [22]).

Now Theorem 5 implies

$$
E_{x}\left(\int_{0}^{t} \chi_{\triangle_{1}}\left(X_{s}\right) d \tilde{\mathfrak{p}}_{s}^{\varepsilon, m}\right)=0, \quad t \geqq 0, \quad x \in D^{*} .
$$

On the other hand, we have from identity (4.17),

$$
E_{x}\left(\sum_{\sigma_{n}^{\varepsilon} \leqq t} \chi_{\triangle_{1}}\left(X_{\sigma_{n}^{\varepsilon}}{ }^{\varepsilon}\right) \chi_{B_{m}}\left(X_{\sigma_{n}^{\varepsilon}}{ }^{2}\right)\right)=E_{x}\left(\int_{0}^{t} \chi_{\triangle_{1}}\left(X_{s}\right) d \tilde{p}_{s}^{\delta, m}\right),
$$

$x \in D^{*}$ (Lemma 3.2 of [22]). The left hand side of equation (4.20) is, owing to assertions (iii) and (iv) of Lemma 3.7, no other than $E_{x}\left(\mathfrak{p}_{t}^{\left.\varepsilon, B_{m}\right)}\right.$. Therefore, the formulae (4.19) and (4.20) imply $\mathfrak{p}_{t}^{\varepsilon, B_{m}} \approx 0$, and consequently assertion (4.16).

We call the conservative diffusion process $\left\{X_{t}, \mathfrak{F}_{t+}, P_{x}, x \in D_{1}^{*}\right\}$ the reflecting barrier Brownian motion on $D_{1}^{*}=D \cup \triangle_{1}$.

Consider the case when $\partial D$ is of class $C^{3}$. By virtue of Lemma 3.1 (iv), we can find a homeomorphism $\Psi$ from $D \cup \partial D$ onto $D^{*}$ such as $\Psi(x)=x, x \in D$. In this case, $\triangle_{0}$ is empty and so, $D^{*}=D_{1}^{*}$ (see the identity (3.11) and the proof of Lemma 3.1). Set $\dot{X}_{t}=\Psi^{-1}\left(X_{t}\right), t \geqq 0$ and $\dot{P}_{x}=P_{\Psi(x)}, x \in D \cup \partial D$. Theorem 2 and the argument in the paragraph following Theorem 1 now imply

Theorem 6. Suppose that $\partial D$ is of class $C^{3}$. Then, $\dot{X}=\left(\dot{X}_{t}, \dot{P}_{x}, x\right.$ $\in D \cup \partial D)$ is a conservative diffusion process on $D \cup \partial D$ satisfying $\dot{P}_{x}\left(\dot{X}_{t} \in E\right)$ 
$=\int_{E \cap D} \dot{p}(t, x, y) d y, t>0, x \in D \cup \partial D$, for any Borel set $E$ of $D \cup \partial D$. Here, $\dot{p}(t, x, y), t>0, x \in D^{*}, y \in D$ is the fundamental solution of the heat equation $\left(\frac{\partial}{\partial t}-\frac{1}{2} \triangle\right) u(t, x)=0$ with the condition $\frac{\partial}{\partial n_{x}} u(t, x)=0, x \in \partial D$. We call $\dot{\boldsymbol{X}}$ the reflecting barrier Brownian motion on $D \cup \partial D$.

See K. Sato and T. Ueno [21] for another version of $\dot{\boldsymbol{X}}$.

Tokyo University of Education

\section{References}

[1] C. Constantinescu and A. Cornea: Ideale Ränder Riemannscher Flächen, Springer, 1963.

[2] J.L. Doob, Stochastic processes, Wiley, New York, 1953.

[3] E.B. Dynkin: Foundations of the theory of Markov processes, Moscow, 1959. (Russian).

[4] E.B. Dynkin: Markov processes, Moscow, 1963. (Russian).

[5] M. Fukushima: Resolvent kernels on a Martin space, Proc. Japan Acad. 41 (1964), 167-175.

[6] N. Ikeda, K. Sato, H. Tanaka and T. Ueno: Boundary problems in multidimensional diffusion processes, Seminar on Probability, vol. 5, 1960; vol. 6, 1961. (Japanese).

[7] S. Ito: Fundamental solutions of parabolic differential equations and boundary value problems, Japan. J. Math. 27 (1957), 55-102.

[8] K. Ito and H.P. McKean Jr: Diffusion processes and their sample paths, Springer, 1965.

[9] H. Kunita and H. Nomoto: On a method of the compactification in the theory of the Markov process, Seminar on Probability, vol. 14, 1962. (Japanese).

[10] H. Kunita and T. Watanabe: On certain reversed processes and their application to potential theory and boundary theory, J. Math. Mech. 15 (1966), 393-434.

[11] H. Kunita and T. Watanabe: Some theorems concerning resolvents over locally compact spaces, to appear in Proc. of 5-th Berkeley Symposium.

[12] Z. Kuramochi: Mass distributions on the ideal boundaries of abstract Riemann surfaces. II, Osaka Math. J. 8 (1956), 145-186.

[13] R.S. Martin: Minimal positive harmonic functions, Trans. Amer. Math. Soc. 49 (1941), 137-172.

[14] P.A. Meyer: Fonctionelles multiplicatives et additives de Markov, Ann. Inst. Fourier 12 (1962), 125-230.

[15] P.A. Meyer: Probabilités et potentiel, Hermann, Paris, 1966.

[16] C. Miranda: Equazioni alle derivate parziali di tipo ellittico, Springer, 1955.

[17] M. Motoo: The sweeping-out of additive functionals and processes on the boundary, Ann. Inst. Stat. Math. 16 (1964), 317-345.

[18] M. Motoo and S. Watanabe: On a class of additive functionals of Markov processes, J. Math. Kyoto Univ. 4 (1965), 429-469. 
[19] M. Ohtsuka: An elementary introduction of Kuramochi boundary, J. Sci. Hiroshima Univ. Ser A-I, 28 (1964), 271-299.

[20] D. Ray: Resolvents, transition functions and strongly Markovian processes, Ann. of Math. 70 (1959), 43-78.

[21] K. Sato and T. Ueno: Multi-dimensional diffusion and the Markov process on the boundary, J. Math. Kyoto Univ. 4 (1965), 529-605.

[22] S. Watanabe: On discontinuous additive functionals and Levy measures of a Markov process, Japan. J. Math. 34 (1964), 53-70.

[23] D.V. Widder: The Laplace transform, Princeton, 1946. 
